\title{
Frequency-Domain Analysis of the Energy Budget in an Idealized Coupled Ocean-Atmosphere Model
}

\author{
PAige E. MARTin \\ Department of Physics, University of Michigan, Ann Arbor, Michigan \\ BRIAN K. ARBIC \\ Department of Earth and Environmental Sciences, University of Michigan, Ann Arbor, Michigan \\ ANDREw McC. HogG AND ANDREw E. KISS \\ Research School of Earth Sciences and ARC Centre of Excellence for Climate Extremes, \\ Australian National University, Canberra, Australian Capital Territory, Australia \\ JAMES R. MUNROE \\ Department of Physics and Physical Oceanography, Memorial University of Newfoundland, St. John's, \\ Newfoundland and Labrador, Canada \\ JEFFREY R. BLUNDELL \\ Ocean and Earth Science, National Oceanography Centre, University of Southampton Waterfront Campus, \\ Southampton, United Kingdom
}

(Manuscript received 13 February 2019, in final form 2 October 2019)

\begin{abstract}
Climate variability is investigated by identifying the energy sources and sinks in an idealized, coupled, ocean-atmosphere model, tuned to mimic the North Atlantic region. The spectral energy budget is calculated in the frequency domain to determine the processes that either deposit energy into or extract energy from each fluid, over time scales from one day up to 100 years. Nonlinear advection of kinetic energy is found to be the dominant source of low-frequency variability in both the ocean and the atmosphere, albeit in differing layers in each fluid. To understand the spatial patterns of the spectral energy budget, spatial maps of certain terms in the spectral energy budget are plotted, averaged over various frequency bands. These maps reveal three dynamically distinct regions: along the western boundary, the western boundary current separation, and the remainder of the domain. The western boundary current separation is found to be a preferred region to energize oceanic variability across a broad range of time scales (from monthly to decadal), while the western boundary itself acts as the dominant sink of energy in the domain at time scales longer than 50 days. This study paves the way for future work, using the same spectral methods, to address the question of forced versus intrinsic variability in a coupled climate system.
\end{abstract}

\section{Introduction}

The source of low-frequency variability in the climate system is a topic of long-standing interest. In the coupled ocean-atmosphere system, it is difficult to decipher whether low-frequency variability is due to intrinsic processes driven by nonlinear advection, due to forcing

Corresponding author: Paige E. Martin, paigemar@umich.edu from the other fluid, or due to the inherently coupled nature of the ocean-atmosphere system. In other words, is the low-frequency variability in the ocean or atmosphere "free" (intrinsic), "forced" (by the opposing fluid), or intrinsically coupled? This question, with particular interest in showing the existence and importance of low-frequency intrinsic ocean variability, has been addressed by many previous studies (e.g., Dewar 2003; Dijkstra and Ghil 2005; Kravtsov et al. 2006; Hogg and 
Blundell 2006; Berloff et al. 2007a,b; Penduff et al. 2011; Quattrocchi et al. 2012; Sérazin et al. 2015; Huck et al. 2015; Kiss and Frankcombe 2016). Recently there has been interest in diagnosing intrinsic versus forced ocean variability using spectral energy budget techniques, as in Arbic et al. (2012, 2014), O'Rourke et al. (2018), and Sérazin et al. (2018). This paper follows these studies and is the first in a series of works that will address the question of distinguishing between intrinsic, forced, and coupled variability through application of a spectral energy budget diagnostic computed in the temporal frequency domain.

In recent years, it has been shown that ocean mesoscale eddies (on the order of $10-100 \mathrm{~km}$ and larger) play a large role in ocean variability, even at low frequencies corresponding to decadal time scales and longer (e.g., Penduff et al. 2011; Delworth et al. 2012). It thus follows that explicit eddy resolution in ocean models is necessary in order to accurately capture the magnitude and behavior of oceanic variability. Additionally, it has been found that ocean mesoscale activity can have significant influence on the air-sea coupling (e.g., Small et al. 2008; Kirtman et al. 2017), although the exact mechanisms driving such coupling are still an open question. In this work, we investigate the effect of such nonlinearities in the ocean within the context of a simplified, but dynamically rich coupled ocean-atmosphere model, with the goal of analyzing ocean eddy variability and the resulting impact on atmospheric variability across a wide range of time scales.

The importance of oceanic eddies in driving midlatitude, low-frequency ocean variability has long been a topic of interest in idealized models. For instance, Dewar (2003) ran a suite of idealized ocean models with periodic forcing at different time scales, as well as with stochastic forcing, and showed that nonlinearities in the ocean are important in decadal variability when using semirealistic ocean forcing. Specific modes of variability have been identified in various idealized ocean models. A turbulent oscillator mode was proposed by Berloff et al. (2007b) with a rough time scale of 12 years, and has been found in other quasigeostrophic models as well (e.g., Shevchenko et al. 2016). This mode is characterized by changes to the magnitude and position of the oceanic jet in the western boundary current separation region. Another such mode is the gyre mode (Simonnet and Dijkstra 2002), which has been characterized by dynamical systems approaches to studying variability, with the identification of bifurcations in the system, and is typically associated with 7- to 8 -yr time scales. In this paper, the goal is not necessarily to identify specific modes of variability in the ocean or atmosphere (although we do point out one particular low-frequency mode of variability) but rather to diagnose the frequency dependence of the different processes in the model, and to diagnose the sources and sinks of energy at each frequency.

The spectral energy budget technique used in this paper was pioneered by Saltzman (1957), and was later simplified and extended into the frequency domain by Hayashi (1980). The terms in the spectral energy budget, as opposed to, for example, energy spectra, are useful for identifying the sources and sinks of energy in a system. Because the terms are defined as cross spectra (Hayashi 1980), they are the product of terms and thus reveal the contribution that each term plays in the energy budget of the fluid. The sign of the spectral energy budget term indicates whether the term inputs energy into or extracts energy from the system, and the relative magnitudes of each term correspond to the amount of energy being added to or removed from the system. That is, a term that displays a large positive magnitude compared to other terms at a certain frequency is said to dominate the input of energy at that frequency. The properties of spectral energy budget analysis in both wavenumber and frequency space are investigated and discussed in detail in Morten (2015). We refer to this framework as a "spectral energy budget" but note that the terms in this budget have also been called "spectral energy transfers" in earlier literature. Integrals of individual spectral energy budget terms from a given frequency to infinity have also been used in the literature, referred to as "spectral fluxes" (Arbic et al. 2014; Sérazin et al. 2018). In this work, we choose to display our results as spectral energy budgets, as they are more visually straightforward in revealing which terms input or extract energy at specific frequencies.

The spectral energy budget analysis (and the spectral flux technique) has been used in numerous other studies prior to this one, although primarily in the wavenumber domain (e.g., Salmon 1978, 1980; Hua and Haidvogel 1986; Larichev and Held 1995; Scott and Wang 2005). Sheng and Hayashi (1990a,b), as well as Arbic et al. (2012, 2014), apply the spectral energy budget/flux method to investigate the existence of energy cascades in frequency space, and in particular to demonstrate the existence of a temporal equivalent of the inverse energy cascade (that energy at short time scales gets transferred to longer time scales). Two more recent studies (O'Rourke et al. 2018; Sérazin et al. 2018) have also used frequency-domain spectral energy budgets to study coupled ocean-atmosphere behavior.

More specifically, Sheng and Hayashi (1990a,b) and Arbic et al. $(2012,2014)$ apply the frequency-domain spectral energy budget technique to a full energy budget 
of an idealized, homogeneous, two-layer, geostrophic turbulence model, with prescribed baroclinically unstable background flows. They also investigate the surface kinetic energy (KE) advection term in a realistic ocean model, as well as a satellite altimeter product. Sérazin et al. (2018) use the same spectral technique applied to a realistic ocean model, but use two different atmospheric forcing fields, one with minimal time dependence, and one with the full spectrum of atmospheric time scales. By comparing the two model runs with differing atmospheric forcing they are able to draw conclusions about the intrinsic versus forced sources of variability. However, they look only at the ocean surface kinetic energy term driven by nonlinear advection. O'Rourke et al. (2018) also apply frequency-domain spectral energy budget diagnostics but to a realistic fully coupled ocean-atmosphere model, and examine the behavior of the wind stress, in addition to the surface KE advection.

We use the Quasi-Geostrophic Coupled Model (Q-GCM; Hogg et al. 2003), which is a two-way coupled, quasigeostrophic (QG), ocean-atmosphere model. Q-GCM is a regional model, made up of an ocean coupled to a channel atmosphere that we have configured to mimic the North Atlantic Ocean (a double gyre configuration). The atmosphere is a reentrant channel that spans the circumference of Earth, centered at $40^{\circ} \mathrm{N}$. The model is quasigeostrophic, with ageostrophic mixed layers at the interface between the fluids that allow for coupling between the ocean and atmosphere. The model has been previously used for various projects, including by Hogg et al. (2006), who used Q-GCM to study coupled modes of variability, and Farneti (2007), who investigated coupled Rossby waves. In this study, we choose Q-GCM because it is well suited for studying ocean-atmosphere variability, can resolve geostrophic eddies in both the ocean and atmosphere, allows us to calculate each term in a closed energy budget, and is computationally cheap to run compared to many largescale coupled climate models.

As is often the case in climate modeling, there is a large gap between idealized studies [e.g., the doubly periodic ocean-only model in Arbic et al. $(2012,2014)]$ and more realistic studies [e.g., the global, fully coupled, high-resolution GFDL model suite used in O'Rourke et al. (2018)]. The medium complexity of Q-GCM as a coupled, yet still reasonably simplified oceanatmosphere model makes it a perfect bridge between these two sides. This paper thus seeks to fill the gap left open by the previously cited literature: we use a more complicated idealized ocean model than Arbic et al. $(2012,2014)$ and couple it with a fully dynamic (albeit idealized) atmosphere. Our model is complex in that it is dynamically inhomogeneous and is a fully coupled model but, unlike in O'Rourke et al. (2018), Sérazin et al. (2018), and the realistic model in Arbic et al. (2012, 2014), it is simple enough to easily permit explicit calculation of all of the terms in the energy budget. Additionally, to our knowledge, this paper is the first to apply the spectral energy budget analysis to both atmospheric and oceanic terms in a coupled model. To visually highlight the novelty of this work compared to previous research on frequency-domain spectral energy budgets, see the diagram in Fig. 1.

In this paper, we apply the frequency-domain spectral energy budget diagnostic to the energy budget of Q-GCM in order to quantify, as a function of frequency, the relative importance of various terms in the energy budget, including intrinsic nonlinear advection and forcing from each fluid. Furthermore, we investigate different spatial regions in the ocean-along the western boundary and the western boundary current separationthat display distinct dynamics, and compare how the sources and sinks of energy differ from one region to the next. In section 2, we describe our setup of Q-GCM and derive the spectral energy budget equations. In section 3, we discuss the domain-integrated kinetic energy spectra and the spectral energy budget in both the oceanic and atmospheric domains. Spatial maps of terms in the ocean spectral energy budget integrated over defined frequency bands are addressed in section 4 , and in section 5 we regionally integrate the spectral energy budget terms over regions of interesting dynamics. Finally, in section 6, we offer some physical interpretations of the results.

\section{Methods}

\section{a. The Quasi-Geostrophic Coupled Model}

We use Q-GCM version 1.5.0 (Hogg et al. 2014) to investigate the sources and sinks of oceanic and atmospheric variability. We choose to configure Q-GCM as a box ocean, meant to represent a basin such as the North Atlantic Ocean, coupled to a reentrant channel atmosphere. Each fluid consists of three quasigeostrophic vertical layers, as depicted in Fig. 2. We choose three layers as it has been found (e.g., in Hogg et al. 2006) that three layers are necessary and sufficient to produce semirealistic baroclinic instability, and thus yield stronger eddies than, say, in a two-layer configuration. As Fig. 2 shows, the ocean occupies a relatively small portion of the atmospheric domain and so the atmosphere is mostly underlain by land, which acts as a solid boundary. The model is run on a $\beta$ plane, with no bottom topography, and is forced solely by temporally and zonally 


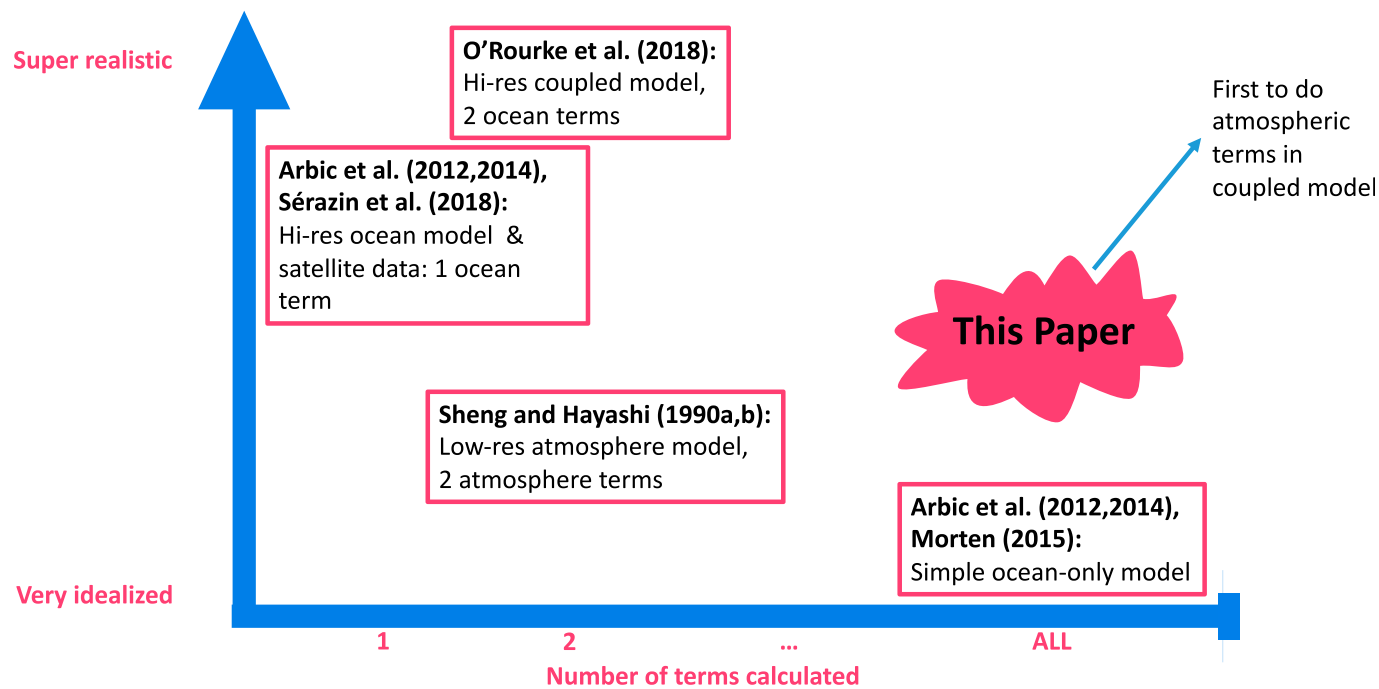

FIG. 1. A graph depicting the novelty of this work in the context of previous research using frequency-domain spectral energy budget analysis. The $y$ axis represents the complexity of the model/dataset and the $x$ axis displays the number of spectral energy budget terms that are explicitly calculated. This work fills a gap left open by the realistic studies that only allow for the calculation of one or two spectral energy budget terms, and the idealized ocean- and atmosphere-only studies.

constant but latitudinally varying solar radiation. The forcing has no temporal variation (i.e., there is not seasonal variation), which ensures that all observed variability is intrinsic to the model.
The ocean-atmosphere coupling in Q-GCM is accomplished through mixed layers that are embedded into the first layers (the layers at the ocean-atmosphere interface) of each fluid. The mixed layers also allow for

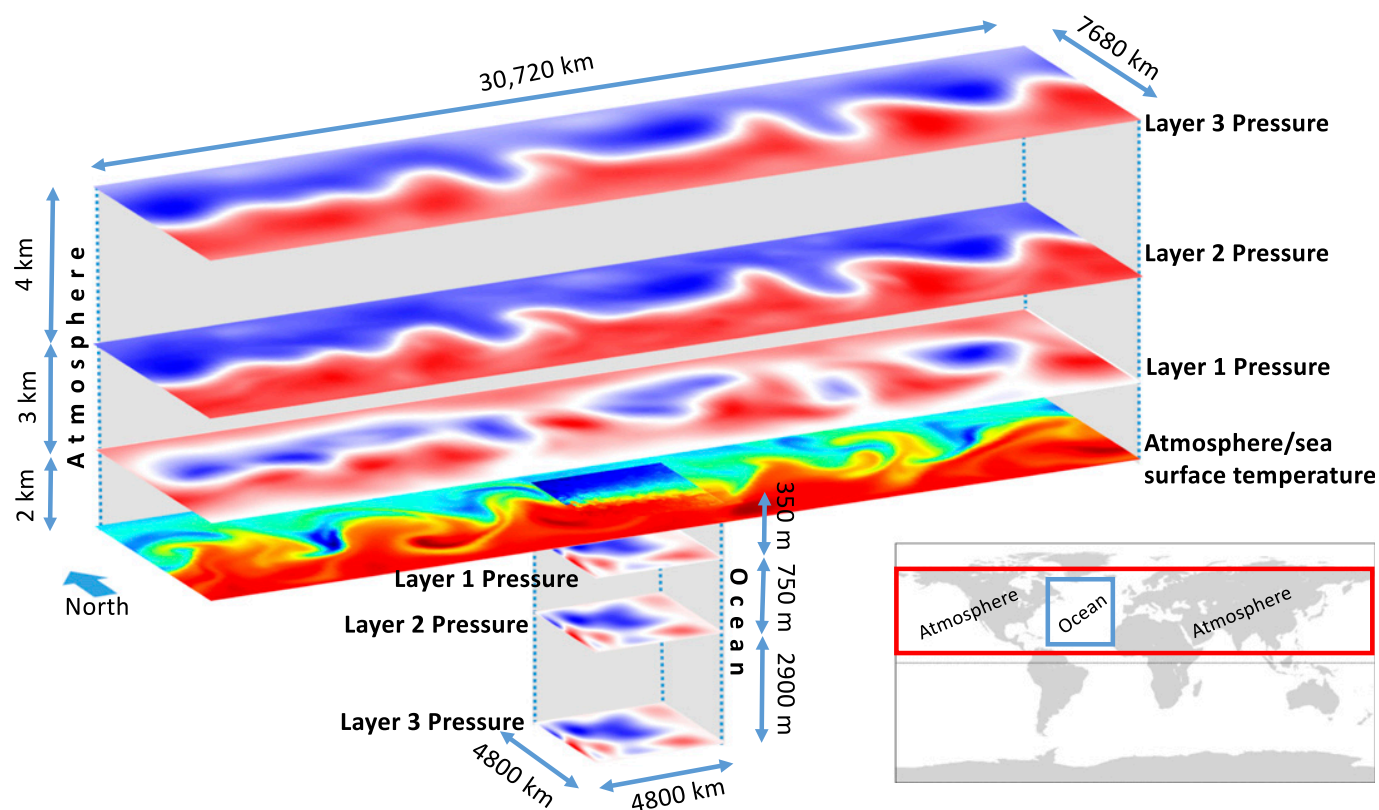

FIG. 2. An exploded view of the quasigeostrophic coupled model setup used in this paper. Layers are shown with snapshots of pressure for each of the three layers in the ocean and atmosphere, and snapshots of surface temperature for each fluid at the ocean-atmosphere interface. Note that the vertical axis is not drawn to scale. The map on the bottom right displays the rough geographical location of the ocean (outlined in blue) and atmosphere (outlined in red) of Q-GCM, and the black horizontal dotted line depicts the location of the equator. 

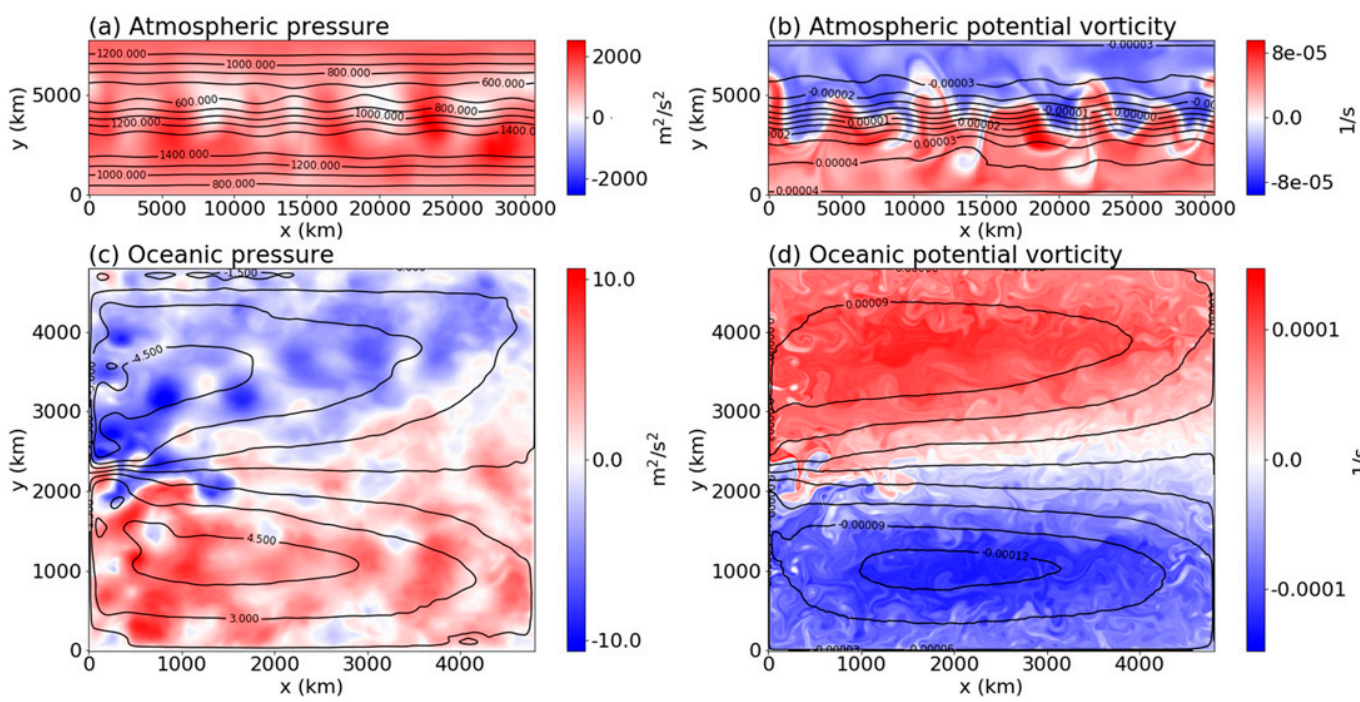

(b) Atmospheric potential vorticity

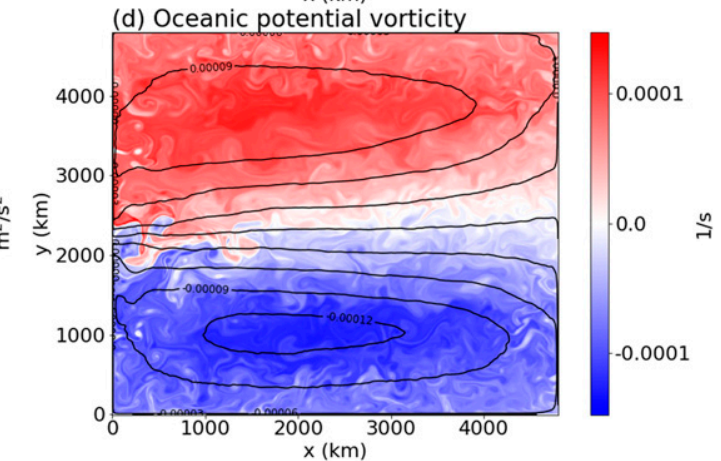

FIG. 3. Q-GCM layer 1 snapshots (in color) and 20-yr averages (black contours) of (a) atmospheric dynamic pressure and (b) potential vorticity, and (c) oceanic dynamic pressure and (d) potential vorticity.

the vertical transport of heat, via parameterized radiation and convection schemes, as well as stress via Ekman pumping (Hogg et al. 2003, 2014).

Snapshots with overlaid average contours of pressure and potential vorticity in both the ocean and atmosphere are shown in Fig. 3. The atmospheric channel in the model displays an eastward jet with eastward propagating waves through the center of the channel, with some westward motions in the northern and southern portions of the domain. The ocean portion of the model shows a double-gyre configuration, with a subtropical gyre and a subpolar gyre, and a strongly eddying western boundary current separation (meant to mimic the Gulf Stream) between the two gyres. This strong current in the ocean roughly aligns with the peak of the atmospheric jet, which occurs where the wind stress curl changes sign.

Following Hogg et al. (2014), the governing equations for Q-GCM's dynamics are written in terms of potential vorticity tendency equations, with potential vorticity $\mathbf{q}$ and pressure $\mathbf{p}$ :

$$
\begin{aligned}
\frac{\partial}{\partial t} \mathbf{q} & =\frac{1}{f_{0}} J(\mathbf{q}, \mathbf{p})+\mathbf{B} \mathbf{e}-\frac{A_{4}}{f_{0}} \nabla^{6} \mathbf{p} \\
\mathbf{q} & =\beta\left(y-y_{0}\right)+\frac{1}{f_{0}} \nabla^{2} \mathbf{p}-f_{0} \mathbf{A p},
\end{aligned}
$$

with Jacobian $J$ defined as

$$
J(\mathbf{q}, \mathbf{p})=\left(\frac{\partial \mathbf{q}}{\partial x}\right)\left(\frac{\partial \mathbf{p}}{\partial y}\right)-\left(\frac{\partial \mathbf{p}}{\partial x}\right)\left(\frac{\partial \mathbf{q}}{\partial y}\right),
$$

where $\mathbf{q}=\left[q_{1}, q_{2}, q_{3}\right], \mathbf{p}=\left[p_{1}, p_{2}, p_{3}\right]$ (with subscripts indicating vertical layer, and layer numbers increasing away from the ocean-atmosphere interface), $t$ is time, $x$ and $y$ are, respectively, the zonal and meridional coordinates, $y_{0}$ is the midlatitude of $40^{\circ} \mathrm{N}, A_{4}$ is the biharmonic viscosity constant, $\nabla$ is the horizontal gradient operator, and we use the $\beta$-plane approximation with $f=f_{0}+\beta\left(y-y_{0}\right)$. Other symbols are defined in Table 1 . In the above equations, $\mathbf{p}$ is the dynamic pressure (pressure divided by the mean density). The matrices $\mathbf{A}$ and $\mathbf{B}$ and vector $\mathbf{e}$ are defined as follows, and superscripts are used to distinguish between ocean $(o)$ and atmosphere $(a)$ :

$$
\begin{gathered}
\mathbf{A}^{o}=\mathbf{A}^{a}=\left[\begin{array}{ccc}
\frac{1}{H_{1} g_{1}^{\prime}} & -\frac{1}{H_{1} g_{1}^{\prime}} & 0 \\
-\frac{1}{H_{2} g_{1}^{\prime}} & \frac{1}{H_{2}}\left(\frac{1}{g_{1}^{\prime}}+\frac{1}{g_{2}^{\prime}}\right) & -\frac{1}{H_{2} g_{2}^{\prime}} \\
0 & -\frac{1}{H_{3} g_{2}^{\prime}} & \frac{1}{H_{3} g_{2}^{\prime}}
\end{array}\right], \\
\mathbf{B}^{o}=-\mathbf{B}^{a}=\left[\begin{array}{cccc}
\frac{f_{0}}{H_{1}} & -\frac{f_{0}}{H_{1}} & 0 & 0 \\
0 & \frac{f_{0}}{H_{2}} & -\frac{f_{0}}{H_{2}} & 0 \\
0 & 0 & \frac{f_{0}}{H_{3}} & -\frac{f_{0}}{H_{3}}
\end{array}\right],
\end{gathered}
$$


TABLE 1. Q-GCM constants.

\begin{tabular}{|c|c|c|}
\hline Parameters & Ocean values & Atmosphere values \\
\hline Basin dimensions & $4800 \mathrm{~km} \times 4800 \mathrm{~km}$ & $30720 \mathrm{~km} \times 7680 \mathrm{~km}$ \\
\hline Number of grid points & $960 \times 960$ & $384 \times 96$ \\
\hline Horizontal grid spacing & $5 \mathrm{~km}$ & $80 \mathrm{~km}$ \\
\hline Approximate latitude-longitude values & $18^{\circ}-62^{\circ} \mathrm{N}, 10^{\circ}-70^{\circ} \mathrm{W}$ & $5^{\circ}-75^{\circ} \mathrm{N}$ \\
\hline Atmosphere indices over ocean & - & $x: 163-223, y: 19-79$ \\
\hline Layer thicknesses $\left(H_{1}, H_{2}, H_{3}\right)$ & $350,750,2900 \mathrm{~m}$ & $2000,3000,4000 \mathrm{~m}$ \\
\hline Mixed layer thicknesses $\left(H_{m}^{o}, H_{m}^{a}\right)$ & $100 \mathrm{~m}$ & $1000 \mathrm{~m}$ \\
\hline Time step & $9 \min$ & $3 \mathrm{~min}$ \\
\hline Bottom Ekman layer thickness $\left(\delta_{\mathrm{ek}}\right)$ & $1 \mathrm{~m}$ & - \\
\hline Reduced gravities $\left(g_{1}^{\prime}, g_{2}^{\prime}\right)$ & $0.015,0.0075 \mathrm{~m} \mathrm{~s}^{-2}$ & $1.2,0.4 \mathrm{~m} \mathrm{~s}^{-2}$ \\
\hline Biharmonic viscosity $\left(A_{4}\right)$ & $2 \times 10^{9} \mathrm{~m}^{4} \mathrm{~s}^{-1}$ & $1.5 \times 10^{14} \mathrm{~m}^{4} \mathrm{~s}^{-1}$ \\
\hline Mean Coriolis parameter-at $40^{\circ} \mathrm{N}\left(f_{0}\right)$ & $9.37456 \times 10^{-5} \mathrm{~s}^{-1}$ & $9.37456 \times 10^{-5} \mathrm{~s}^{-1}$ \\
\hline$y$ derivative of Coriolis parameter $(\beta)$ & $1.75360 \times 10^{-11} \mathrm{~m}^{-1} \mathrm{~s}^{-1}$ & $1.75360 \times 10^{-11} \mathrm{~m}^{-1} \mathrm{~s}^{-1}$ \\
\hline Mixed boundary condition parameter $\left(\alpha_{\mathrm{bc}}\right)$ & 0.2 & 1.0 \\
\hline Density $(\rho)$ & $1000 \mathrm{~kg} \mathrm{~m}^{-3}$ & $1 \mathrm{~kg} \mathrm{~m}^{-3}$ \\
\hline Heat capacity $\left(C_{p}\right)$ & $1 \times 10^{3} \mathrm{~J} \mathrm{~kg}^{-1} \mathrm{~K}^{-1}$ & $4 \times 10^{3} \mathrm{~J} \mathrm{~kg}^{-1} \mathrm{~K}^{-1}$ \\
\hline Layer temperatures $\left(T_{1}, T_{2}, T_{3}\right)$ & $287,282,276 \mathrm{~K}$ & $330,340,350 \mathrm{~K}$ \\
\hline Temperature diffusion coefficients $\left(K_{2}, K_{4}\right)$ & $200 \mathrm{~m}^{2} \mathrm{~s}^{-1}, 2 \times 10^{9} \mathrm{~m}^{4} \mathrm{~s}^{-1}$ & $2.5 \times 10^{4} \mathrm{~m}^{2} \mathrm{~s}^{-1}, 2 \times 10^{14} \mathrm{~m}^{4} \mathrm{~s}^{-1}$ \\
\hline Drag coefficient $\left(C_{D}\right)$ & - & $1.3 \times 10^{-3}$ \\
\hline Mean radiative forcing $\left(\overline{F_{S}}\right)$ & - & $-220 \mathrm{~W} \mathrm{~m}^{-2}$ \\
\hline Radiation perturbation magnitude $\left(F_{S}^{\prime}\right)$ & - & $80 \mathrm{~W} \mathrm{~m}^{-2}$ \\
\hline Adiabatic lapse rate $(\gamma)$ & - & $1 \times 10^{-2} \mathrm{~km}^{-1}$ \\
\hline
\end{tabular}

$$
\begin{aligned}
& \mathbf{e}^{o}=\left[\begin{array}{c}
w_{\mathrm{ek}}^{o} \\
e_{1}^{o} \\
0 \\
\frac{\delta_{\mathrm{ek}}}{2 f_{0}} \nabla^{2} p_{3}
\end{array}\right], \\
& \mathbf{e}^{a}=\left[\begin{array}{c}
w_{\mathrm{ek}}^{a} \\
e_{1}^{a} \\
0 \\
0
\end{array}\right],
\end{aligned}
$$

where the Ekman velocity $w_{\mathrm{ek}}$ is proportional to the vertical component of the curl of the wind stress $\tau$ :

$$
\begin{aligned}
& w_{\mathrm{ek}}^{o}=\mathbf{k} \cdot \frac{\nabla \times \tau^{o}}{f_{0}}, \\
& w_{\mathrm{ek}}^{a}=\mathbf{k} \cdot \frac{\nabla \times \tau^{a}}{f_{0}},
\end{aligned}
$$

where $\mathbf{k}$ is the vertical unit vector. The oceanic and atmospheric stresses differ only by a factor of the density ratio. Stress is calculated using relative wind, such that the wind stress depends on the difference between the atmospheric and oceanic surface velocities (where velocities over land are set to zero). The ocean and atmosphere entrainments $e_{1}$ in Eqs. (5) and (6) are defined as

$$
\begin{aligned}
& e_{1}^{o}=-\frac{T_{m}^{o}-T_{1}^{o}}{2\left(T_{1}^{o}-T_{2}^{o}\right)} w_{\mathrm{ek}}^{o}, \\
& e_{1}^{a}=\frac{F_{m}^{\uparrow}+F_{1}^{\downarrow}}{\rho^{a} C_{p}^{a}\left(T_{2}^{a}-T_{1}^{a}\right)} .
\end{aligned}
$$

The formulation for the time-varying mixed layer temperature $T_{m}^{o}$ and the heat fluxes $F_{m}^{\uparrow}$ and $F_{1}^{\downarrow}$ can be found in Hogg et al. (2014). The values of the constant layer temperatures, as well as constants $H_{k}$ (layer heights), $\delta_{\mathrm{ek}}$ (bottom Ekman layer thickness), $g_{i}^{\prime}$ (reduced gravity values at each interface $i$ ), and $C_{p}$ (heat capacity), are given in Table 1, along with descriptions and values of other constants used in the model.

The ocean and atmosphere have resolutions of 5 and $80 \mathrm{~km}$, respectively. The time step is $3 \mathrm{~min}$ in the atmosphere and $9 \mathrm{~min}$ in the ocean, and the output is in daily snapshots. The Q-GCM parameters of reduced gravity, layer thicknesses, Coriolis parameters, and basin size have been tuned to mimic the North Atlantic Ocean double-gyre circulation and the atmosphere at those same latitudes. We set a constant value of pressure along each of the ocean boundaries that varies in time, and use a mixed condition on the nonperiodic boundaries in the atmosphere, such that there is a different value of pressure at the north and south boundaries. For derivatives of pressure on all solid boundaries, we use a partial slip condition based on the value of a constant $\alpha_{\mathrm{bc}}$ (included in Table 1). A more thorough explanation of the model's mixed boundary conditions can be found in 
appendix C of Hogg et al. (2014). The parameters were tuned by Jeff Blundell and Chris Wilson (personal communication, 19 May 2015), using the same procedure as described in section 2.1 of Wilson et al. (2015), which in turn was based on Flierl (1978).

The model is initialized from a state of radiative balance, with mixed layer temperatures given by Eqs. (D.7) and (D.9) of Hogg et al. (2014). The atmospheric layer pressures for layer $n>1$ are then adjusted to give the interface displacement given in Eq. (D.8) of Hogg et al. (2014). This will give an initial (zonal) geostrophic flow in the upper layers of the atmosphere. The ocean starts from rest. The model is then spun up by introducing the latitudinally varying solar radiation that gets deposited directly into the ocean mixed layer and land points. Radiation is then reemitted by the land points into the atmospheric mixed layer at relatively short time scales (on the order of days), while radiation re-emitted from the ocean mixed layer is much slower due to the ocean's large heat capacity. We run the model for 50 years before considering it to be in a statistically steady state, after which we run the model for 400 years. Figure 4 shows the layer kinetic energy (per unit area) for each fluid in the 50-yr spinup phase. The atmosphere in Fig. 4a reaches a steady state much sooner (around 1 year) than the ocean in Fig. 4b (around 10 years), but both fluids are clearly in a steady state by the end of the 50 -yr spinup phase. For more detailed information about Q-GCM in general, we refer the reader to Hogg et al. $(2003,2014)$.

\section{b. Spectral energy budget equations}

We analyze the Q-GCM output using a frequency-domain spectral energy budget technique, as used in Saltzman (1957), Hayashi (1980), Arbic et al. (2012, 2014), Morten (2015), O'Rourke et al. (2018), and Sérazin et al. (2018). To derive the spectral energy budget equation, we first need to obtain the energy tendency equations for both the ocean and the atmosphere. To obtain the energy tendency equation, we start with the Q-GCM governing equations and follow the standard procedure in geostrophic energetics (e.g., section 5.6.2 in Vallis 2006) whereby the potential vorticity tendency equation is multiplied by pressure. With a term given by the product of pressure and the Laplacian of pressure, by integration by parts over the domain, we get an equation proportional to the time derivative of the square of the gradient of pressure. In geostrophy, the square of the pressure gradient is proportional to the square of velocity, and hence we obtain an energy equation. We perform the previous steps in the frequency domain to yield an equation for the spectral energy budget in each fluid.

We start with the governing equations given in Eq. (1) in the previous section, and rewrite them in terms of pressure only: (a) Average atmosphere KE over the 50-year spin-up
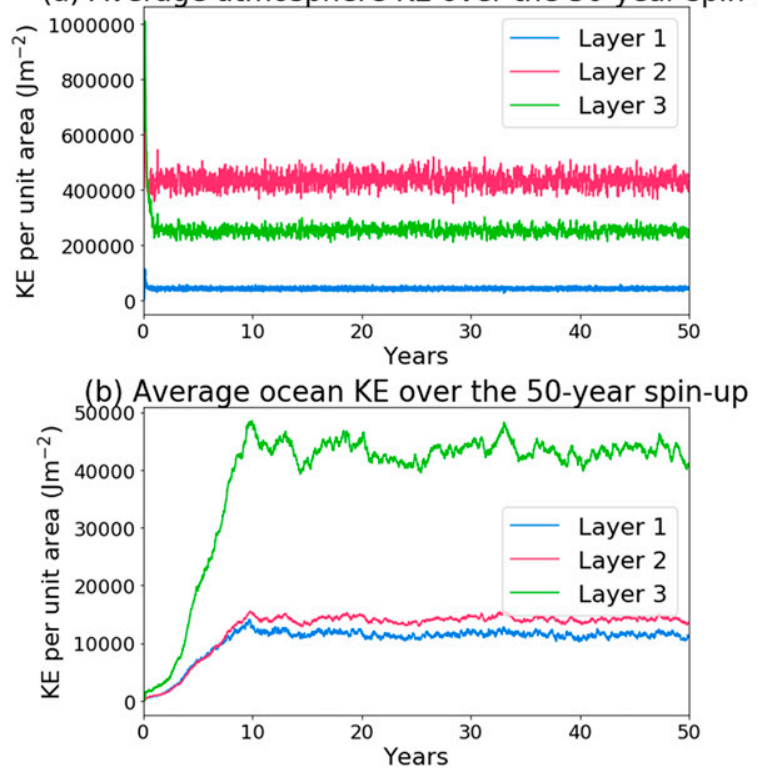

FIG. 4. Average layer kinetic energy (per unit area) for the 50-yr spinup period in (a) the atmosphere and (b) the ocean. The atmosphere spins up in roughly 1 year, whereas the ocean takes about 10 years to fully spin up.

$$
\begin{aligned}
\nabla^{2}\left(\frac{\partial}{\partial t} \mathbf{p}\right)-f_{0}^{2} \frac{\partial}{\partial t} \mathbf{A p}= & -\beta \frac{d \mathbf{p}}{d x}+\frac{1}{f_{0}} J\left(\nabla^{2} \mathbf{p}, \mathbf{p}\right)-f_{0} J(\mathbf{A} \mathbf{p}, \mathbf{p}) \\
& +f_{0} \mathbf{B e}-A_{4} \nabla^{6} \mathbf{p} .
\end{aligned}
$$

To derive the remainder of the calculations, we continue by writing out only the first-layer ocean equation, and consequently omit the zero subscript. All other layers in both fluids follow nearly identical steps. Rewriting Eq. (9) for ocean layer 1 yields

$$
\begin{aligned}
\nabla^{2}\left(\frac{\partial}{\partial t} p_{1}\right)= & \frac{f_{0}^{2}}{H_{1} g_{1}^{\prime}} \frac{\partial}{\partial t}\left(p_{1}-p_{2}\right)-\beta \frac{d p_{1}}{d x}+\frac{1}{f_{0}} J\left(\nabla^{2} p_{1}, p_{1}\right) \\
& +\frac{f_{0}}{H_{1} g_{1}^{\prime}} J\left(p_{2}, p_{1}\right)+\frac{f_{0}^{2}}{H_{1}}\left(w_{\mathrm{ek}}-e_{1}\right)-A_{4} \nabla^{6} p_{1} .
\end{aligned}
$$

We now take the Fourier transform of this equation in time:

$$
a=\sum_{\omega} \hat{a}(x, y, \omega) e^{i \omega t},
$$

where $a$ is an arbitrary function, and $\hat{a}$ is its Fourier transform. If we rewrite the terms in this form, and divide both sides by $e^{i \omega t}$, we are left with 


$$
\begin{aligned}
i \omega \nabla^{2} \widehat{p_{1}}= & i \omega \frac{f_{0}^{2}}{H_{1} g_{1}^{\prime}}\left(\widehat{p_{1}}-\widehat{p_{2}}\right)-\beta \frac{d \widehat{p_{1}}}{d x}+\frac{1}{f_{0}} J \widehat{\left(\nabla^{2} p_{1}, p_{1}\right)} \\
& \left.+\frac{f_{0}}{H_{1} g_{1}^{\prime}} J \widehat{\left(p_{2}, p_{1}\right.}\right)+\frac{f_{0}^{2}}{H_{1}}\left(\widehat{w_{\mathrm{ek}}}-\widehat{e_{1}}\right)-A_{4} \nabla^{6} \widehat{p_{1}} .
\end{aligned}
$$

To obtain an equation for the energy of the system, we first multiply Eq. (12) by $\widehat{p}_{1}$ *, where the star indicates the complex conjugate. We also multiply the complex conjugate of Eq. (12) by $\widehat{p_{1}}$, and then add the two equations together and divide by two, collecting terms using the property $\left(a^{*} b+b^{*} a\right) / 2=\operatorname{Re}\left[a^{*} b\right]$ (where $\operatorname{Re}[x]$ is the real part of $\left.x\right):$

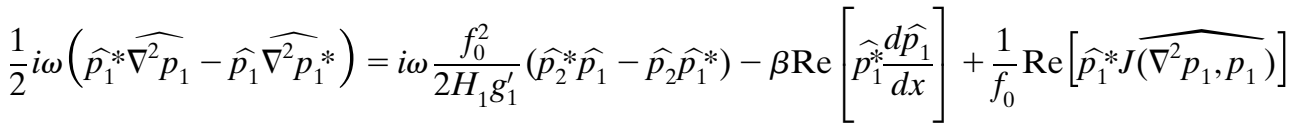

$$
\begin{aligned}
& +\frac{f_{0}}{H_{1} g_{1}^{\prime}} \operatorname{Re}\left[\widehat{p}_{1}^{*} J\left(\widehat{\left.p_{2}, p_{1}\right)}\right]+\frac{f_{0}^{2}}{H_{1}} \operatorname{Re}\left[\widehat{p}_{1}^{*} \widehat{w_{\mathrm{ek}}}\right]-\frac{f_{0}^{2}}{H_{1}} \operatorname{Re}\left[\widehat{p}_{1}^{*} \widehat{e_{1}}\right]-A_{4} \operatorname{Re}\left[\widehat{p}_{1}^{*} \widehat{\nabla^{6} p_{1}}\right] .\right.
\end{aligned}
$$

The same steps are followed for the remainder of the layers to produce equations similar to Eq. (13) for every layer in both the ocean and the atmosphere. To obtain thickness-weighted equations, we multiply each layer equation by the constant $H_{n} /\left(H_{1}+H_{2}+H_{3}\right)=$ $H_{n} / H_{\text {tot }}$, with $n$ denoting layer. Since the system is in steady state, we can assume that the time derivative of energy averaged over long time periods (the term on the left-hand-side of the equation) is zero. We then sum all of the layers together to yield energy budget equations in the ocean and in the atmosphere. We note that the first term on the right-hand side of Eq. (13) sums to zero across all layers in the thickness-weighted sum.

The final step is to take the area integral to find an equation in terms of frequency only. The $\beta$ term drops out upon domain integration, and we are left with the final spectral energy budget equation for the ocean (where we have multiplied through by $1 / f_{0}^{2}$ to have correct units of $\mathrm{m}^{2} \mathrm{~s}^{-2}$ ):

$$
\begin{aligned}
& 0=\iint\{\underbrace{\frac{1}{f_{0}^{3} H_{\text {tot }}} \sum_{n=1}^{3} H_{n} \operatorname{Re}\left[\widehat{p}_{n}^{*} J\left(\nabla^{2} \widehat{p_{n}, p_{n}}\right)\right.}_{\text {Advection of KE, layer } n}]+\underbrace{\frac{1}{f_{0} g_{1}^{\prime} H_{\text {tot }}} \sum_{i=1}^{2} \operatorname{Re}\left[\widehat{\left(p_{i+1}-p_{i}\right)} \widehat{J\left(p_{i+1}, p_{i}\right)}\right]}_{\text {Advection of PE, interface } i} \\
& +\underbrace{\frac{1}{H_{\text {tot }}} \operatorname{Re}\left[\widehat{p}_{1}^{*} \widehat{w_{\mathrm{ek}}}\right.}_{\text {Wind stress }}]-\underbrace{\frac{1}{H_{\text {tot }}} \operatorname{Re}\left[\left(\widehat{p_{2}-p_{1}}\right)^{*}\right.}_{\text {Buoyancy forcing }} \widehat{e_{1}}]-\underbrace{\frac{\delta_{\mathrm{ek}}}{2 f_{0} H_{\mathrm{tot}}} \operatorname{Re}\left[\widehat{p_{3}} * \widehat{\nabla^{2} p_{3}}\right]}_{\text {Bottom drag }}+[\text { biharmonic viscosity] }\} d x d y .
\end{aligned}
$$

One can see that the advection of potential energy depends on the difference between layer pressures, which corresponds to the height perturbation between levels in a QG model, and hence why the term describes potential energy. Similarly, the advection of kinetic energy term contains a product of pressure and (a derivative of) the Laplacian of pressure, which yields a kinetic energy when integrated over an area. We also mention that Ekman transport is not included in the advection terms, but the wind stress and buoyancy terms depend on Ekman velocity. Note that we drop the word "nonlinear" throughout this paper when referring to nonlinear advection.

The spectral energy budget equation for the atmosphere is very similar: 


$$
\begin{aligned}
& 0=\iint\{\underbrace{\frac{1}{f_{0}^{3} H_{\text {tot }}} \sum_{n=1}^{3} H_{n} \operatorname{Re}\left[\widehat{p_{n}^{*}} \widehat{J\left(\nabla^{2} p_{n}, p_{n}\right)}\right]}_{\text {Advection of KE, layer } n}-\underbrace{\left.\frac{1}{f_{0} g_{1}^{\prime} H_{\text {tot }}} \sum_{i=1}^{2} \operatorname{Re}\left[\widehat{\left(p_{i+1}-p_{i}\right.}\right) * \widehat{J\left(p_{i+1}, p_{i}\right)}\right]}_{\text {Advection of PE, interface } i} \\
& +\underbrace{\frac{1}{H_{\text {tot }}} \operatorname{Re}\left[\widehat{p_{1}} * \widehat{w_{\text {ek }}}\right]}_{\text {Bottom drag }}-\underbrace{\frac{1}{H_{\text {tot }}} \operatorname{Re}\left[\left(\widehat{p_{2}-p_{1}}\right)\right.}_{\text {Buoyancy forcing }} * \widehat{e_{1}}]+[\text { biharmonic viscosity] }\} d x d y .
\end{aligned}
$$

Note the different definitions behind the terms titled "bottom drag" between the ocean and atmosphere. For details about integrating over the domain and the handling of boundary conditions we refer the reader to appendix E of Hogg et al. (2014).

These equations are applied to the Q-GCM output for periods of 100 model years, in order to capture decadal variability in the ocean. With a 400 -yr model run, we average over seven 100 -yr time series with 50-yr overlapping windows to get better statistical significance, particularly in the lower frequencies. Before the Fourier transform is applied to the data, we apply a detrending and a Tukey window function (with a taper-to-constant ratio of $20 \%$ ) in the time domain of the data to correct its nonperiodicity.

These spectral energy budget equations allow us to determine whether each term is a source or sink of energy at a given frequency. It should be noted that this work exclusively addresses the frequency dependence of ocean-atmosphere variability. By detrending the data (removing the time mean), we do not investigate how the terms in Eqs. (14) and (15) contribute energy into or out of the time mean. The spectral energy budget framework does not reveal how or whether energy is transferred from one term to another, or from the mean to the variable part of the flow. Our goal is to understand the relative contributions from each term to the frequency-domain energy budget within the time-variable flow.

\section{Domain-integrated results}

\section{a. Time-domain energy budgets}

Before presenting our frequency-domain results, we first plot the area-integrated time-domain energy budgets. Shown in Fig. 5 are the energy budgets for the atmosphere (Fig. 5a) and the ocean (Fig. 5b) for 1 year of the Q-GCM run, 65 years after the 50 -yr spinup phase. The residuals (the sums of all terms) are shown in gray, and are very close to zero, indicating that the energy budgets are closed (i.e., that energy is conserved over time). The terms in the energy budgets were calculated according to the method outlined in appendix E of Hogg et al. (2014). The biharmonic viscosity terms in both the oceanic and atmospheric energy budgets are an order of magnitude smaller than the other terms in the budgets, and so we do not include the terms in the spectral energy budget analysis. Additionally, two cells at the nonperiodic boundaries in both fluids are masked in the calculation of the (time domain and frequency domain) energy budget terms to ease with derivative calculations. However, the plotted residuals are sufficiently close to zero at each frequency that we deem the aforementioned effects to be negligible.

\section{b. Kinetic energy spectra}

The domain-integrated kinetic energy spectra of each layer in both the atmosphere and ocean are shown in Fig. 6. The black dashed lines on the right-hand side of each plot have a slope of -2 . This line is included to compare our results with section 8.5 in Tennekes and Lumley (1972), which shows that, analogous to the classic Kolmogorov argument in wavenumber space, frequency-domain spectra in 2D turbulent flows follow a power law with exponent -2 , assuming homogeneous and isotropic turbulence. [Note that although we are using the term "spectra" throughout this paper, we have in fact plotted spectral density, i.e., the energy spectra divided through by the spacing between frequencies; see section 8.6.2 of Stull (1988).]

In the atmosphere (Fig. 6a), layer 3 follows a slope of -2 at high frequencies, with layer 2 exhibiting a slightly shallower slope, and layer 1 being even shallower. We speculate that this shallower-than-expected behavior in the first layer stems from the ageostrophic buoyancy terms in the atmospheric mixed layer interacting with the first layer of the atmosphere. At a frequency corresponding to a period of 20 days, we observe a peak in all of 

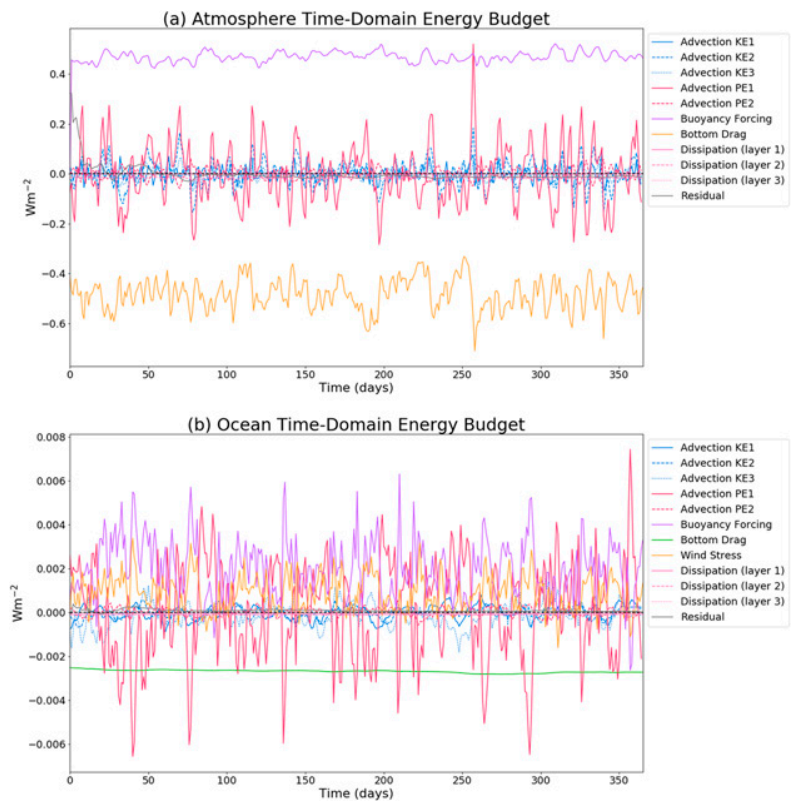

FIG. 5. Domain-integrated time-domain energy budgets in the (a) atmosphere and (b) ocean for 1 model year, 65 years after the spinup phase.

the spectra that is roughly at the time scale it takes for waves to travel zonally around the atmospheric channel. At all lower frequencies the shapes of the energy spectra in each layer are nearly identical. It is worth noting that these spectra have been weighted by layer thickness. Thus, we see that layer 2 harbors the most energy of any layer, which is to be expected since the greatest velocities are found in layer 2. Layer 1 spectral values are an order of magnitude smaller than either layer 2 or 3 , which is likely due to bottom drag over ocean and land points.

In the ocean (Fig. 6b), we observe the same slope of -2 in the high frequencies corresponding to about 30 days and shorter. In the ocean, however, it is layer 1 that is the most energetic, while layer 3 is the least energetic. Neither of these results is a surprise, since the first ocean layer is in contact with the dynamic atmosphere, and the third ocean layer is in contact with bottom drag. Some obvious features of the ocean spectra are the large, narrow peaks in the high frequencies, present in all of the layers. These peaks are due to barotropic Rossby wave basin modes (Longuet-Higgins 1964; LaCasce 2002) that are excited due to the geometry of the Q-GCM ocean basin (in this case, a square). Each narrow peak corresponds to the different number of nodes in each excited 2D standing wave. We calculated the theoretical frequencies at which the basin modes should occur in the Q-GCM ocean basin, as given by Eq. (5) in LaCasce (2002), and found that the locations of the high-frequency peaks in the spectra shown in Fig. $6 \mathrm{~b}$ are consistent with the theory to within $2 \%$, and their spatial patterns match the theoretical
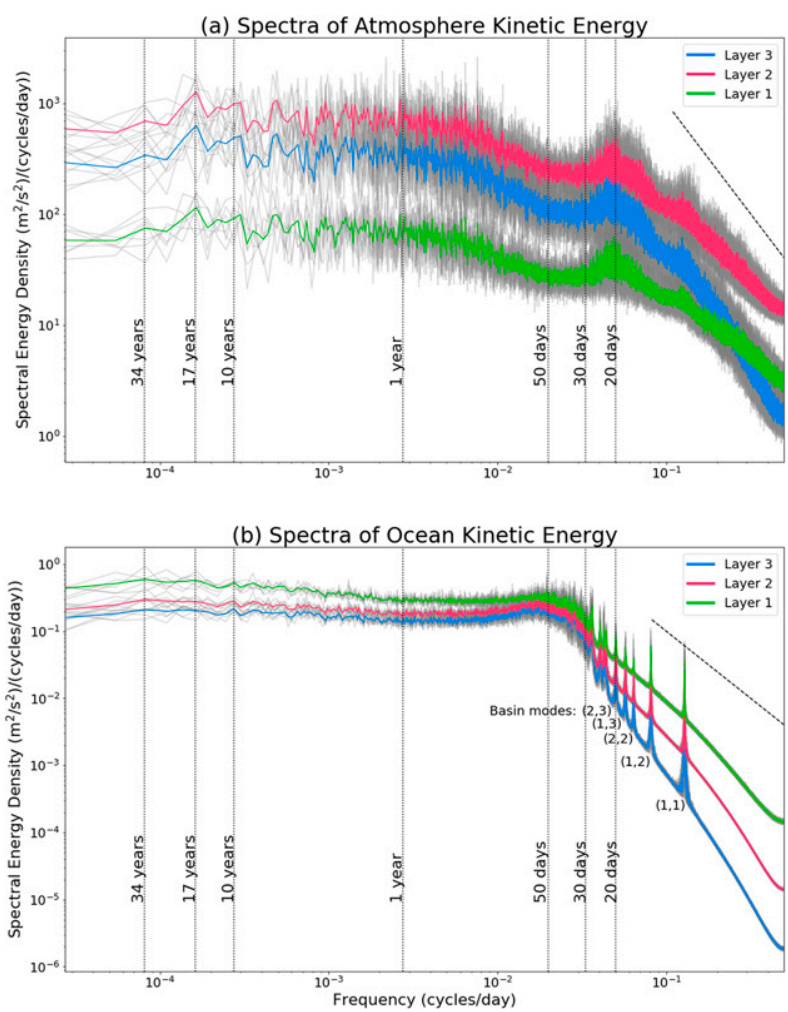

FIG. 6. Domain-integrated kinetic energy spectra of the three layers (a) in the atmosphere and (b) in the ocean. The gray lines are of each 100-yr analysis, and the colored lines show the average over seven 100-yr periods. The basin mode peaks are labeled in (b) with their corresponding wavenumbers $(m, n)$. The dashed line in each figure has the theorized 2D-turbulent regime slope of -2 .

expectation (not shown). The first five modes are labeled in Fig. $6 \mathrm{~b}$ as $(m, n)$, where $m$ and $n$ are the wavenumbers in the $x$ and $y$ direction, respectively. We refer the reader to LaCasce (2002) for a deeper understanding of these basin modes in geostrophic turbulence models.

\section{c. Spectral energy budget analysis}

The domain-integrated spectral energy budgets in both the ocean and atmosphere are shown in Fig. 7. The spectral energy budgets are closed in the same way as the physical-space energy budgets (Fig. 5), and the residual is plotted for both fluids to show that the terms balance at each frequency.

\section{1) AtMosphere}

In Fig. 7a, which shows the domain-integrated spectral energy budget of the atmosphere, we observe that the second layer kinetic energy advection (KE2) is the dominant source of energy at all frequencies corresponding to time scales of around 20 days and longer. The same term is also a sink of energy at higher frequencies. This behavior of taking energy out of the system at high frequencies, and 
(a) Atmosphere Spectral Energy Budget

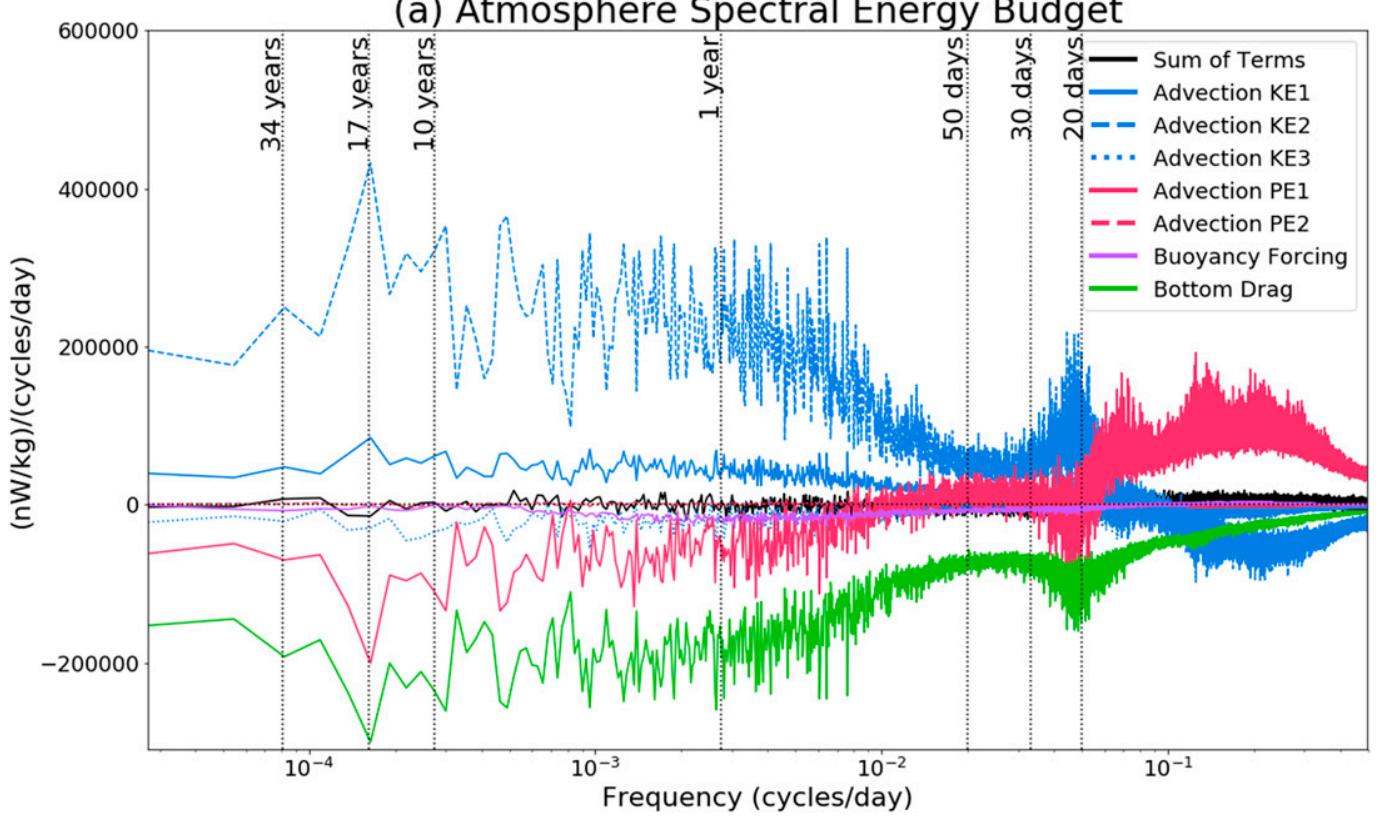

(b) Ocean Spectral Energy Budget

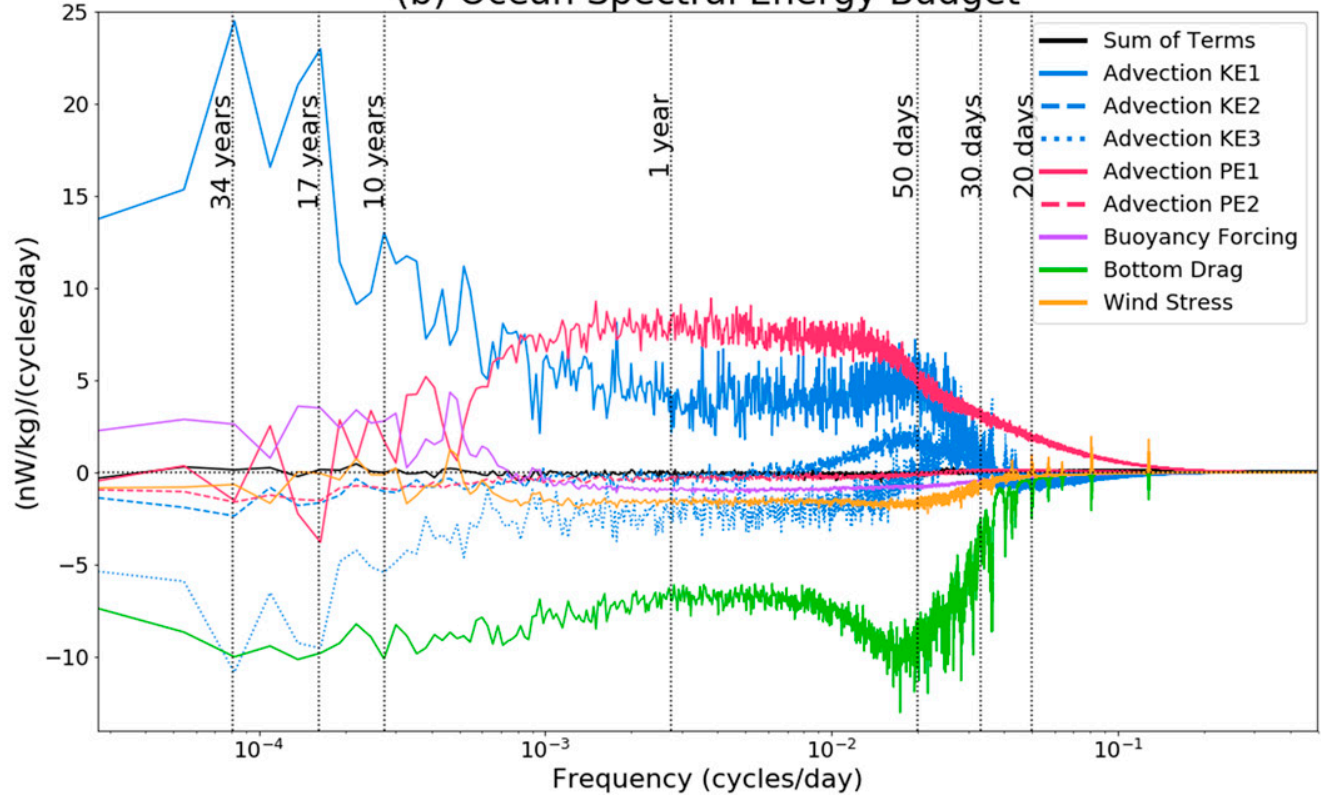

FIG. 7. Domain-integrated spectral energy budgets in the (a) atmosphere and (b) ocean. Each curve shown is the average over seven 100-yr periods, and the residual (the sum of all of the terms) in each fluid is shown in black. Note the different vertical scales between the two plots.

adding energy to the system at low frequencies is consistent with the "inverse temporal cascade" seen in Arbic et al. (2012, 2014), Morten (2015), O’Rourke et al. (2018), and Sérazin et al. (2018), where energy is transferred from high to low frequencies. We see this same inverse cascade behavior in the advection of first layer kinetic energy (KE1) but with a much smaller magnitude which, as mentioned previously, is likely due to the higher velocities in atmospheric layer 2 compared to layer 1 . We also note that there is a low-frequency peak in KE2, and to a lesser degree KE1, at a time scale of 17 years, which we also observe in the ocean. The third layer KE advection term (KE3) is not a significant contributor to the overall energy budget of the atmosphere.

The potential energy advection at the first interface (PE1) roughly opposes KE2 (except in the 20-100-day 
band), with positive values at high frequencies (shorter than around 20 days) in which it also opposes bottom drag, and mostly negative at lower frequencies. This result suggests the presence of a forward cascade of energy: energy is transferred out of the low frequencies and deposited into the high frequencies. This behavior is consistent with Arbic et al. (2014), who found that the $\mathrm{KE}$ and $\mathrm{PE}$ advection terms almost perfectly mirrored one another in their idealized ocean-only model. In turn, the mirroring of frequency-domain PE and KE energy budget terms is consistent with the classical paradigm of wavenumber-domain spectral energy budgets given in Steinberg et al. (1971) and Salmon (1980). The small, though nonzero, magnitude of PE2 suggests that it plays a very small role in the transfer of energy in the system, and we will thus not discuss the term in any more detail. The primary sink of energy in the atmosphere is the bottom drag. This is to be expected, as this term is designed to extract energy out of the system. At very high frequencies, though, it is KE2 that removes the most energy from the system.

The buoyancy term appears to play a very small role in the transfer of energy across all frequencies, but we note that the buoyancy term involves the momentum and heat fluxes at the ocean-atmosphere interface. Because the oceanic domain is so much smaller than the atmospheric domain, the domain-integrated buoyancy term is indeed quite small. However, in Fig. 8 we plot the spectral energy budget of the atmospheric energy terms for the portion of the atmospheric domain that is directly above the ocean, and it is clear that the buoyancy term plays a much larger role in energy transfer in this region. Specifically, the buoyancy term appears to be a sink of energy in the atmosphere at all frequencies, and a particularly significant energy sink in the short and midrange periods up through around 6 years, beyond which it is very close to zero. Other than a shift toward lower frequency in the PE1 zero crossing and a slightly smaller magnitude at the lowest frequencies in KE2, the behavior of the other atmospheric terms does not change qualitatively over the ocean when compared to the entire atmospheric domain. We recognize that these integrals in Fig. 8 do not extend to the domain boundaries, and so strictly speaking cannot be interpreted as energy terms of the region over the ocean, but we refer to them as "energy" for brevity as they show the contribution of this region to the energy budget integrated over the whole channel.

\section{2) OCEAN}

Figure $7 \mathrm{~b}$ shows the domain-integrated spectral energy budget for the ocean portion of Q-GCM. The KE1 term is the dominant term among the layers in the ocean,

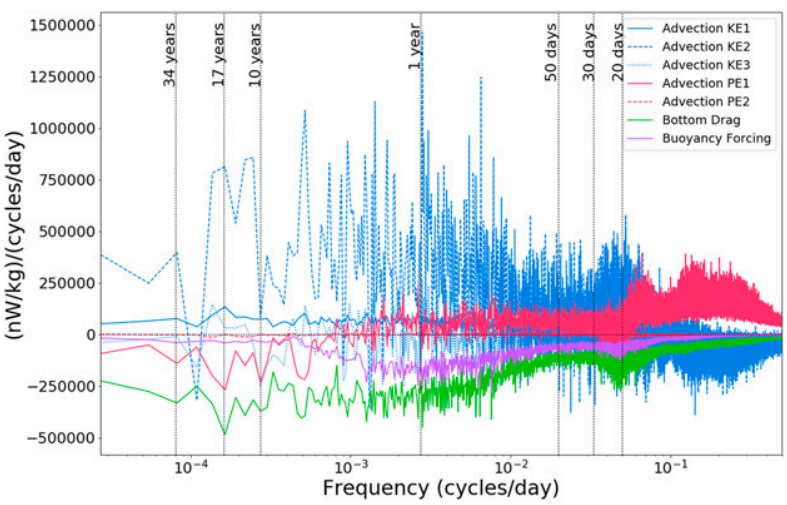

FIG. 8. Area-integrated spectral energy budget in the portion of the atmospheric domain that is directly above the ocean. Each curve shown is the average over seven 100 -yr periods.

contrary to the layer-2-dominated atmosphere KE advection. This can be explained by the highest ocean velocities being in ocean layer 1 , whereas the highest atmospheric velocities are in layer 2. Like the shape of KE2 in the atmosphere, the shape of the ocean's KE1 term with negative values at high frequencies (time scales less than about 30 days) and positive at lower frequencies implies the existence of the "inverse temporal cascade." KE1 advection is the dominant source of energy at low frequencies, from around a time scale of two years and longer. At time scales longer than around 12 years, there is a noticeable increase in the magnitude of KE1, with two peaks corresponding to time scales of roughly 17 and 34 years. Spectral peaks at nearly the exact same frequencies were also found in Hogg et al. (2006) in the first layer ocean height and sea surface temperature fields. The $17-\mathrm{yr}$ peak also coincides with a peak in KE2 at the same time scale in the atmosphere. We revisit and offer some physical insight on these low-frequency peaks in section 5 .

KE2 and KE3 are also negative at high frequencies in the ocean, becoming positive around 30 days. However, they both quickly become negative again for all low frequencies. This spectral shape with positive values in the midrange and negative values in both high and low frequencies may indicate that, in layers 2 and $3, \mathrm{KE}$ cascades from both low and high frequencies to the midrange frequencies. KE3 advection is particularly interesting at time scales longer than around 12 years, when its magnitude roughly matches that of the bottom drag, as they work together to balance the large lowfrequency energy input from KE1. We will look more into KE3's role as a large sink in the ocean's energy budget in section 5 .

PE1 shows a very different shape than in the atmosphere. It is positive at nearly all frequencies, except at the lowest of the frequency range (longer than 12 years), 
when the term appears to oscillate around zero. The mostly positive values tell us that PE1 is a major source of energy in the ocean at all frequencies up to around 12 years. This behavior differs from both the atmosphere and the behavior seen in Steinberg et al. (1971), Salmon (1980), and Arbic et al. (2014), as it does not appear to indicate a forward cascade, which would mirror the inverse cascade of the first layer KE. The behavior of PE1 advection is discussed further in section 5. Due to its small magnitude, PE2 is not considered a significant energy source or sink in the ocean in this system.

As expected, and as in the atmosphere, the bottom drag term is negative (removing energy) at all frequencies and mirrors KE1 at time scales longer than about 30 days. Shorter than 30 days, bottom drag is mostly compensated by positive PE1 values. At high frequencies, we again observe several sharp peaks due to the barotropic Rossby wave basin modes. In the spectral energy budget, the basin modes appear negative in the bottom drag term and are balanced by the wind stress, suggesting that the basin modes are excited by wind variability at their resonant frequency and have their amplitude limited by bottom drag.

The wind stress in the ocean is negative across nearly the entire frequency domain (except between around 5 and 20 years), implying that the wind stress is removing energy at nearly all time scales. This result may appear counterintuitive, as atmospheric wind is considered to be a major forcing of oceanic variability. However, our spectral energy budget plots show the effects of the anomalous wind-that is, what remains after the mean wind contribution has been removed. Thus, the variations in wind stress appear to be removing energy at nearly all frequencies in the domain, albeit at a relatively small amplitude compared to other terms. This damping of eddy energy by anomalous winds is consistent with the results of other studies, including O'Rourke et al. (2018) and von Storch et al. (2007). It may also be consistent with work by Renault et al. (2016), who demonstrate that the use of relative winds to calculate stress in a coupled ocean-atmosphere model can cause the "killing" of ocean eddies, thus acting as a sink of energy in the ocean.

The buoyancy term in Fig. $7 \mathrm{~b}$ appears to be a small sink of energy at frequencies exceeding those corresponding to time scales of four years, but a source of energy at lower frequencies. This buoyancy term is where the mixed layer temperatures enter into the energy budget. Since the magnitude of buoyancy is small compared to other terms, we will not discuss it much further in this paper.

To help understand the behavior behind the domainintegrated spectral energy budgets, we will look at the spectral energy budget terms at individual grid points, before domain integration is performed, in the next section. There are three primary questions about terms in the spectral energy budget in the ocean that are especially interesting: 1) Why is PE1 positive across nearly all frequencies? 2) Why does PE1 not counteract KE1? 3) Why is KE3 such a large sink of energy at low frequencies?

\section{Spatial maps of the ocean spectral energy budget}

To get a better intuition for these spectral energy budget terms, we examine their spatial distribution. In the above calculations, we integrated over the $(x, y)$ domain to yield a closed spectral energy budget as a function of frequency. To examine regional behavior, we now plot the spatial pattern of specific terms integrated over frequency bands. We refer to these plots as spatial maps of the spectral energy budget, and they are functions of spatial axes $x$ and $y$, as well as the limits of the frequency band one integrates over. We aim to be careful with terminology here, since the spatial integration in the previous section allowed for the cancellation of some of the terms in the equations. For instance, when we refer to the spatial map of kinetic energy advection, we are in fact referring to the spatial map of the contribution to the kinetic energy advection that remains after spatial integration (i.e., the terms that do not cancel upon domain integration). The purpose of these spatial maps is to help us understand the terms that we observe in the domain-integrated spectral energy budgets, particularly the ocean's PE1, KE1, and KE3. We note that this work follows that of Arbic et al. (2012, 2014), who investigate only the full-domain integrated spectral energy budget. However, they utilize a doubly periodic QG turbulence model that displays only one dynamical regime, and thus the spatial maps would be of less physical significance.

Several spatial maps of spectral energy budget terms for the ocean are shown in Fig. 9. From left to right, the columns show PE1, KE1, and KE3. The top row displays the domain-integrated spectral energy budget terms, with highlighted frequencies indicating the frequency band over which we average to create the spatial maps in the rest of the rows. The frequency bands were chosen to demonstrate the spatial distribution across low frequencies (180 days to 100 years; highlighted in blue and shown in row 2), midrange frequencies (60-90 days, highlighted in red, shown in row 3 ), and high frequencies (18-20 days, highlighted in green, shown in row 4). Note that in many cases there is significant cancellation between regions of opposite sign in Fig. 9 to give the integrated values in Fig. $7 b$. 
을
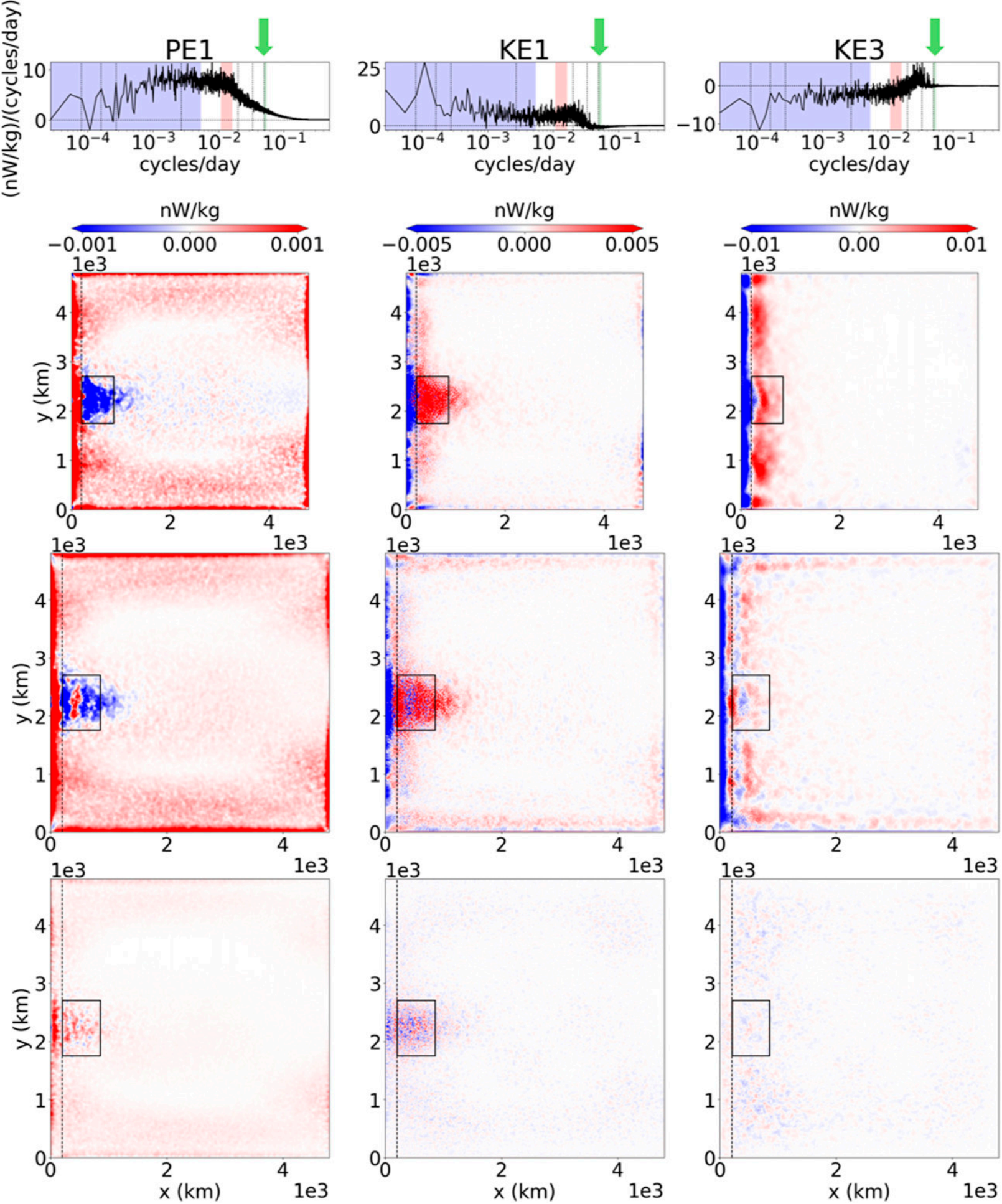

FIG. 9. Spatial maps of ocean spectral energy budget terms for (left) layer-1 PE advection, (middle) layer-1 KE advection, and (right) layer-3 KE advection. (top row) Domain-integrated spectral energy budget term, indicating the three frequency ranges that are averaged over (second row) low frequencies (shaded in blue), (third row) middle frequencies (shaded in red), and (bottom row) high frequencies (shaded in green and indicated with a green arrow). Note the different color bar scales across columns. 
In the first two columns of Fig. 9, the KE1 and PE1 terms show a very clear signature from the western boundary current separation region, which shows up as a triangular-shaped protrusion from the western boundary near the middle latitude of the domain. This triangular region is distinguishable due to the high magnitudes of spectral energy values across all frequency bands, as well as large-scale solid-color patches in the low- and midfrequency bands. Interestingly, if we compare the spatial distribution of the signs of the values between KE1 and PE1, we see that they are mostly opposites of one another. In the low-frequency band, KE1 shows positive values in the western boundary current separation, with PE1 showing negative values in the same region. This opposition is even more pronounced in the middle frequencies with the appearance of a narrow, north-southoriented band of negative values (blue) in KE1 and positive values (red) in PE1 in the middle of the triangular western boundary current separation region of opposite sign. Although the amplitudes differ, we argue that this mirroring in sign between the KE1 and PE1 terms is an indication that there is a distinct oppositional relationship between KE1 and PE1, as found in Steinberg et al. (1971), Salmon (1980), and Arbic et al. (2014), but that these relationships are visible only in certain dynamical regions.

Another region that displays the same kind of opposing behavior is along the western boundary of the ocean domain. Especially pronounced in the lowfrequency band, the western boundary appears to be mostly negative in KE1 and mostly positive in PE1, indicating that KE1 is a sink and PE1 a source in the western boundary. The midfrequency band displays some of this behavior, as well, but it is not easily seen in the high frequencies. However, the western boundary appears to play a very large role in the advection of third layer KE, unlike the current separation region, which is only barely visible in KE3. In the low-frequency band of KE3, we observe strong negative values along the western boundary wall. The middle and high frequencies show noisier signatures, but still display larger magnitudes along the western boundary, relative to the rest of the domain.

There is also a noticeable difference in the spatial scales that are present across frequency bands, particularly in the KE terms displayed in Fig. 9. In the highfrequency band, the small scales are apparent due to the small alternating patches of positive and negative values. In the mid- and low-frequency bands, small-scale features are still present, but with a larger-scale background signature. It therefore appears that large scales occur preferentially at lower frequencies, but that small scales are present at all frequencies. This preference for broader scales to occur at lower frequencies may help demonstrate why an inverse cascade in temporal space has been shown to accompany the more well-known inverse cascade in wavenumber space. This frequencydependent difference in spatial scale is also present in the PE1 term, but to a much lesser degree.

\section{Regional area-integrated ocean spectral energy budgets}

By visual inspection, we split the ocean domain into regions, based on the distinctive spatial signatures in both the western boundary and current separation regions discussed in the previous section. In this section, we will therefore discuss the ocean as the sum of three parts: the western boundary current separation (CS; indicated by the black rectangle in Fig. 9), the western boundary (WB; the portion to the left of the dotted vertical line in Fig. 9), and the remainder of the domain (ROD). Figure 10 displays the area-integrated plots for each of these three regions, which were made following the same steps as in Fig. 7, by taking the average of seven 100 -yr-long time series, and then averaged over the respective regions. Unlike in Fig. 7, these regional spectral energy budgets do not each sum to zero, since they are not integrated across the entire domain. The nonzero residuals in these regions can be explained by the fact that, for a subregion of the domain, the fluxes coming through the boundaries prevent the terms we are looking at from adding to zero.

The spectral energy budget in the CS is shown in Fig. 10a. KE1 is the clear dominant source of energy throughout nearly the entire frequency range, corresponding to time scales from about 20 days up through 100 years. Furthermore, it appears that the only significant sources of energy come from the KE advection terms in each layer, with nearly all other terms negative throughout most of the frequency range, corresponding to time scales beyond around 20 days. PE1 plays the largest role as a sink, particularly at low frequencies. This plot shows that what we observed in the first two columns of Fig. 9 with the opposing role of KE1 and $\mathrm{PE} 1$, is true for the majority of frequencies in the CS. However, their magnitudes are drastically different, and they thus do not completely balance one another, as Arbic et al. (2014) observed in their doubly periodic QG ocean model. Here we see that the KE advection terms are of far greater magnitude than the PE advection terms, with KE1 nearly 6 times the magnitude of PE1.

The WB spectral energy budget (Fig. 10b) shows nearly the reverse of what we observed in the CS. The $\mathrm{KE}$ advection terms are all negative at low frequencies (beyond 50 days), while the PE1 term is positive at all 


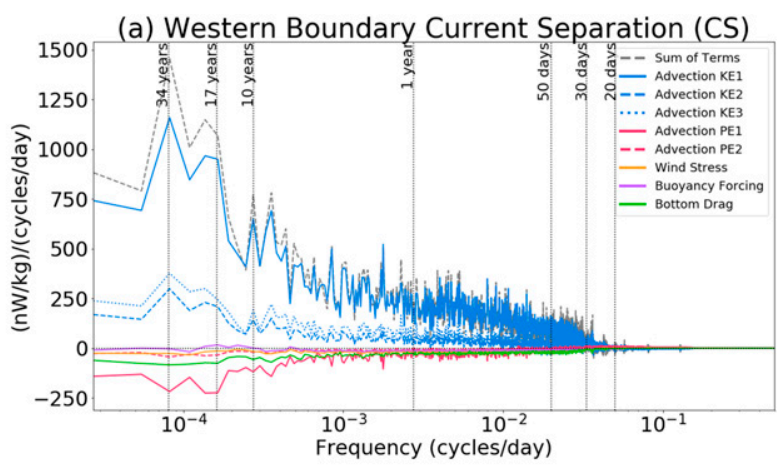

(b) Western Boundary (WB)
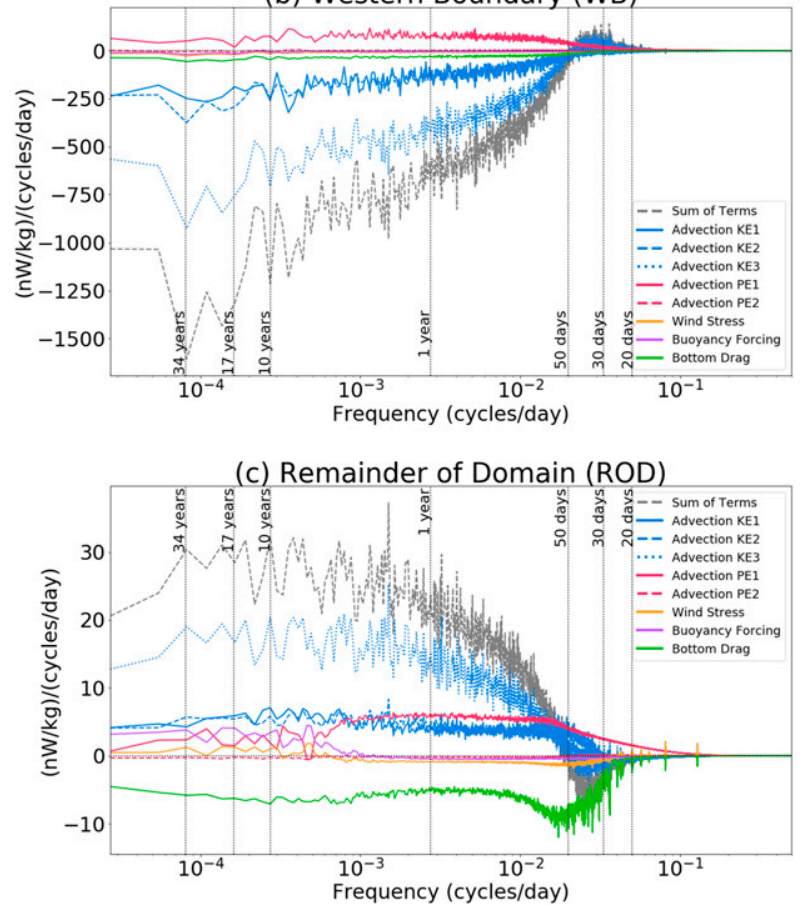

FIG. 10. Regionally area-integrated spectral energy budgets in (a) the western boundary current separation (CS), (b) the western boundary (WB), and (c) the rest of the domain (ROD). Each curve shown is the average over seven 100 -yr periods, and the dashed gray line shows the sum of all the terms in each region. Note that the vertical scales differ between each plot, in order to best illustrate all of the terms in each region.

frequencies. So, we still observe oppositional behavior at low frequencies between the KE and PE1 advection terms, but with their roles reversed: KE advection (dominated by KE3) is a very large sink of energy in the $\mathrm{WB}$, whereas PE1 is a significantly smaller, but still the largest, source of energy in the region. One noticeable difference in the western boundary is that there is a pronounced positive range of $\mathrm{KE}$ advection at frequencies corresponding to time scales shorter than around 50 days, which we do not see being opposed by PE1 (PE1 remains positive at all frequencies). Comparatively, the bottom drag, wind stress, and buoyancy do not play significant roles in either the CS or WB regions.

The area-integrated spectral energy budget for the ROD region is shown in Fig. 10c. We include this plot mostly to show the behavior of the ocean terms outside of the two dynamically interesting regions of the CS and WB. The third-layer KE advection is the largest energy source at frequencies corresponding to 60 days and longer. This is not unexpected outside of the surface eddying regions, given that layer 3 is the thickest ocean layer. Here, though, the KE terms do not overwhelmingly dominate the energy budget nearly as much as in the other two regions, with all terms roughly within one order of magnitude. At all frequencies, the bottom drag extracts the most energy from the region, and is the only significant sink of energy at time scales longer than around 5 years. In contrast to the CS and WB regions, the wind stress is positive at time scales greater than around two years. We suggest that this may be due to the fact that the ROD region is less eddy-dominated (Wilson 2016). The barotropic Rossby basin modes are most obvious in this region, as expected given that the ROD region encompasses the majority of the ocean domain.

These regional spectral energy budget plots also reveal the location of the large low-frequency energy input via $K E 1$, featuring the two peaks at 17 and 34 years that we observed in the full-domain spectral energy budget plot in Fig. 7b. These two peaks in KE1 are most pronounced in the CS region (Fig. 10a), with smaller, but still noticeable, peaks in the other KE advection terms as well. This means that there is a large source of energy at low frequencies in the system coming from KE1 in the CS region. In Fig. 7b, KE3 displays the same two peaks but with negative magnitudes, thus indicating a large sink of energy at low frequencies. Figure 10b suggests that the low-frequency energy input from KE1 in the CS region is being extracted from the system mostly by KE3 along the western boundary, and to a lesser extent by the other KE advection terms also in the WB region.

The gray dashed lines in each plot of Fig. 10 are the sums of the terms and indicate the overall sign of the energy budget term in each region, thus revealing the regions that are overall sources or sinks of energy for the entire system. The CS and ROD regions are primarily sources of energy, whereas WB is a very large sink of energy overall. Furthermore, the overall magnitudes of the CS and WB regions at low frequencies are very similar, further suggesting that the energy input in the CS region (mostly by KE1) is removed in the WB region (mostly by KE3). We can therefore conclude that in the Q-GCM ocean, the primary sink of energy occurs in the WB region. This result is consistent with Zhai et al. (2010), who also identify the western boundary as 
an important sink of ocean eddies. We can go further to suggest that the primary energy source of that energy appears to be in the CS region, with a smaller amount of energy input into the system in the ROD region.

After splitting the ocean into these three different dynamical regions, it becomes apparent that averaging over the entire ocean domain masks a great deal of variability within the different dynamical regimes. It is clear in Fig. 10 that the KE advection terms, in particular, are drastically different depending on the region, and cancel out a lot of their behavior when integrated. For example, KE3 is a large source of energy throughout most of ROD, but is cancelled out by the even larger negative magnitudes in WB. In the full domain, this cancellation results in an average negative value of KE3 at low frequencies, and completely masks the fact that it is positive in both the CS and ROD, and the primary source of energy in the latter. With all of these regional differences, we therefore emphasize the importance of considering regions of differing dynamics when interpreting spectral energy budgets so as to not mask the different behaviors in each region. By using "energy" in this and the previous section, we remind the reader that we are only discussing the contributions to the basin integral. There are other terms in the energy budget that remain prior to domain integration that were not considered here.

\section{Summary and discussion}

We have used the spectral energy budget (or spectral transfer) framework of Arbic et al. $(2012,2014)$ in the frequency domain to investigate the energy variability in the quasigeostrophic coupled model (Hogg et al. 2003). Through a combination of area-integrated spectral energy budgets and spatial maps of frequency-averaged spectral energy budget terms, we have diagnosed the dominant energetic source and sink terms in the ocean and atmosphere in the frequency domain, and have identified dynamically distinct oceanic regions that display strikingly different behavior of energy variability.

Our analysis differs from previous work in several ways:

- We use a model that is both more complex than the idealized, doubly periodic, dynamically homogeneous models used in Arbic et al. (2012, 2014), but simpler than the realistic models used by Arbic et al. $(2012,2014)$, Sérazin et al. (2018), and O'Rourke et al. (2018), which thus allows for richer dynamics, while still explicitly solving for each term in the closed energy budget.

- We use frequency-domain (and not wavenumber) spectral energy budgets, as in Arbic et al. (2012,
2014), O'Rourke et al. (2018), and Sérazin et al. (2018), but different from the majority of earlier literature.

- We are the first study (as far as we know) to apply the spectral energy budget technique to atmospheric terms in a fully coupled model.

- We extend our analysis into lower frequencies, up to 100 years, to compare the differences in variability at high versus low frequencies. With specific interest in climate variability, we considered 100 years to be the minimum amount of time to see decadal variability-a particularly important time scale of variability in the ocean. This is in contrast to the 3-20-yr ranges used in Arbic et al. (2012, 2014) and O'Rourke et al. (2018) and the realistic model/altimetry calculations in Arbic et al. (2012, 2014), and is more in line with the longer, multidecadal model records used in Sérazin et al. (2018).

In this paper, we have made several observations about the behavior of ocean and atmosphere variability:

- Nonlinear advection of KE1 is the dominant source of energy in the ocean at low frequencies (time scales $>3$ years), while nonlinear advection of KE2 is the dominant energy source in the atmosphere at nearly all frequencies (time scales $>20$ days).

- PE1 and KE1 spectral energy budget terms show opposite behavior in the area-averaged atmosphere, but not in the area-averaged ocean. The western boundary current separation (CS) and western boundary (WB) regions, however, do display this classical opposite behavior (Steinberg et al. 1971; Salmon 1980).

- The WB region is a large overall sink of ocean energy at time scales larger than about 50 days, dominated by the KE advection terms (mostly KE3), while the CS region is an overall source of ocean energy, also dominated by the KE advection terms (mostly KE1). Their overall energy budget signs and magnitudes almost perfectly oppose one another, strongly suggesting that there is a low-frequency input of energy in the CS region that is extracted by the WB.

- The 17- and 34-yr peaks of KE1 are seen as energy sources in the CS, suggesting that there is important variability at these time scales in the western boundary current separation.

- The KE terms display larger-scale spatial structures in the low frequencies, but smaller-scale spatial structures are found at all frequencies. This preference for large scales at lower frequencies may help explain the existence of a frequency-domain inverse cascade of $\mathrm{KE}$ alongside the classical inverse cascade in the wavenumber domain (e.g., Salmon 1980; Scott and Wang 2005). 
With the above observations from our analysis, we offer some preliminary physical interpretations of the spectral energy budgets. As mentioned previously, this work is the first in a series, in which we document the methodology and log results from the energy budget of a fully coupled system. In future work, we will apply the same spectral technique to the temperature variance equations to examine the frequency dependence of the heat fluxes and temperature advection explicitly, and then repeat our analysis for partially coupled and decoupled configurations of the model. By comparing the results from both the energy and temperature variance budgets across the different coupling variations, we expect to identify whether it is intrinsic nonlinear advection, forcing from the other fluid, or intrinsic coupling that dominates the low-frequency variability in the oceanic and atmospheric components of the coupled climate system.

In this study, we have shown that the ocean sources and sinks in the strongly eddying regions of the CS and the WB are driven primarily by the nonlinear advection of kinetic energy. This result implies that, in our idealized model, aside from explicit time-mean contributions (which are inherently removed in our spectral analysis method), the ocean eddies and jets are the largest driver of energy transfer in and out of the ocean. These ocean motions at low frequencies, corresponding to time scales of 50 days up to 100 years, appear to mostly be sourced in the CS (and ROD for the third layer) and extracted via the WB. Since the CS region is dominated by KE1 and the WB by KE3, our results suggest that ocean eddies generated near the surface of the ocean (and possibly also westward-propagating Rossby waves) hit the western boundary of the domain, where their energy is transported downward, and are ultimately dissipated in the bottom layer along the western boundary. This finding is consistent with Zhai et al. (2010), who also found that the western boundary is a hot spot for ocean eddy dissipation, although the exact physical mechanism responsible for removing eddy energy along the western boundary remains an open question.

The two sharp, low-frequency peaks of KE1 in the CS region (Fig. 10a) are difficult to explain physically based solely on the methods used here. It is interesting, however, that these peaks are not obvious in the ocean KE spectra shown in Fig. $6 \mathrm{~b}$, thus indicating that these spectral energy budget terms identify special behavior of the system beyond just looking at spectra. As mentioned previously, spectral peaks at nearly identical time scales as we have seen in this work (17 and 34 years) were found by Hogg et al. (2006) also in a coupled run of Q-GCM. In their case, these spectral peaks were observed in the spectra of first mode Hilbert empirical orthogonal functions [see Hogg et al. (2006) for full details] of ocean interface height and sea surface temperature. The fact that these peaks have been observed in Q-GCM in both the current study and in Hogg et al. (2006) implies that these Q-GCM configurations have significant variability at these time scales $(17$ and 34 years, and more generally at time scales longer than 12 years). We suggest that this may be caused by a lowfrequency meander in the western boundary current separation (which mimics the Gulf Stream in this work), but we expect the forthcoming work to shed more light on the source of these low-frequency peaks.

The fact that our analysis has highlighted the eddying regions of the model (the WB and CS) as the most interesting and relevant to the energy budget may help extend some results from other studies. Penduff et al. (2011) and Sérazin et al. (2015) both found that the lowfrequency variability in the active eddying regions, namely the western boundary currents, is mostly due to intrinsic dynamics. In the current work, the KE advection terms dominate the eddying regions of the $\mathrm{WB}$ and $\mathrm{CS}$ at nearly all frequencies, with largest signatures in the low frequencies. These KE advection terms can be thought of as the coupled intrinsic behavior of the ocean in our setup. We are calling it "coupled intrinsic" since the nonlinear advection of $\mathrm{KE}$ is an inherently oceanic process (and so intrinsic to the ocean), but in a coupled regime it is difficult to determine the overall driver of the oceanic $\mathrm{KE}$ advection. In the sense that $\mathrm{KE}$ advection is an indicator of intrinsic behavior, our results are consistent with Penduff et al. (2011) and Sérazin et al. (2015), as well as Sérazin et al. (2018) and Arbic et al. (2012, 2014), who found that nonlinear KE advection was the largest source of energy at low frequencies in their respective models, regardless of eddy activity. Our results can go a step further to say that the primary role of $\mathrm{KE}$ advection in low-frequency oceanic variability, especially in the eddying regions, still exists in a fully coupled system. That is, the dynamic coupling with the atmosphere does not (completely) dampen the dominance of $\mathrm{KE}$ advection in the maintenance of lowfrequency variability in the eddy-active regions of the ocean. In fact, we also observe peaks in KE2 at the same time scales (although more prominently at 17 years). If the peaks in ocean KE advection are in fact intrinsic, the similarly peaked behavior in the atmosphere could be an indication that the ocean forces the atmosphere at these time scales. This will be further investigated with the comparison of decoupled and partially coupled Q-GCM runs, when we will be able to better decipher the exact source of low-frequency variability in these regions. Furthermore, future work with explicit mixed layer temperature dependence will help us to diagnose how these eddying regions might affect atmospheric 
variability, which has been a topic of interest (e.g., Minobe et al. 2008; Small et al. 2008).

This is the first paper in a series that uses frequencydomain spectral energy budgets to study the energy and temperature variance budgets in Q-GCM, run under different coupling configurations, with the goal of identifying intrinsic versus forced versus coupled behavior. This particular work is designed to demonstrate that the spectral energy budget framework in the frequency domain is a powerful tool for determining which terms are sources and sinks of energy in the climate system, and at what locations, organized by frequency. We have highlighted specific oceanic regions and time scales in this simplified model that may be of particular importance to understanding the sources of variability in more complex models and in the climate system as a whole. The results from this paper could help us to better understand how our current climate behaves, and also help us identify the spatial and temporal scales that may be especially robust or vulnerable to climate change.

Acknowledgments. The authors thank the following individuals for their help and insight: Amanda O'Rourke, Aidan Heerdegen, and Bruno Deremble. Special thanks are due to William Dewar for many useful discussions over the course of this project. The authors also wish to recognize the reviewers whose comments significantly improved this manuscript. This material is based upon work supported by the National Science Foundation Graduate Research Fellowship under Grant DGE 1256260. PEM also acknowledges the associated Graduate Research Opportunities Worldwide fellowship to conduct research at the Australian National University. Q-GCM and the analysis codes were run on the National Computational Infrastructure (NCI), which is supported by the Australian Government. The codes are written in Python with the Pangeo environment. PEM and BKA acknowledge support from NSF Grants OCE-0960820 and OCE-1351837, and the University of Michigan African Studies Center and M-Cubed program, the latter supported by the Office of the Provost and the College of Literature, Science, and the Arts.

\section{REFERENCES}

Arbic, B. K., R. B. Scott, G. R. Flierl, A. J. Morten, J. G. Richman, and J. F. Shriver, 2012: Nonlinear cascades of surface oceanic geostrophic kinetic energy in the frequency domain. J. Phys. Oceanogr., 42, 1577-1600, https://doi.org/10.1175/JPO-D-110151.1.

_, M. Müller, J. G. Richman, J. F. Shriver, A. J. Morten, R. B Scott, G. Sérazin, and T. Penduff, 2014: Geostrophic turbulence in the frequency-wavenumber domain: Eddy-driven low-frequency variability. J. Phys. Oceanogr., 44, 2050-2069, https://doi.org/10.1175/JPO-D-13-054.1.

Berloff, P., W. Dewar, S. Kravtsov, and J. McWilliams, 2007a: Ocean eddy dynamics in a coupled ocean-atmosphere model. J. Phys. Oceanogr., 37, 1103-1121, https://doi.org/10.1175/JPO3041.1.

— A. M. Hogg, and W. Dewar, 2007b: The turbulent oscillator: A mechanism of low-frequency variability of the wind-driven ocean gyres. J. Phys. Oceanogr., 37, 2363-2386, https://doi.org/ 10.1175/JPO3118.1.

Delworth, T. L., and Coauthors, 2012: Simulated climate and climate change in the GFDL CM2.5 high-resolution coupled climate model. J. Climate, 25, 2755-2781, https://doi.org/ 10.1175/JCLI-D-11-00316.1.

Dewar, W. K., 2003: Nonlinear midlatitude ocean adjustment. J. Phys. Oceanogr., 33, 1057-1082, https://doi.org/10.1175/ 1520-0485(2003)033<1057:NMOA > 2.0.CO;2.

Dijkstra, H. A., and M. Ghil, 2005: Low-frequency variability of the large-scale ocean circulation: A dynamical systems approach. Rev. Geophys., 43, RG3002, https://doi.org/10.1029/ 2002RG000122.

Farneti, R., 2007: Coupled interannual Rossby waves in a quasigeostrophic ocean-atmosphere model. J. Phys. Oceanogr., 37, 1192-1214, https://doi.org/10.1175/JPO3061.1.

Flierl, G. R., 1978: Models of vertical structure and the calibration of two-layer models. Dyn. Atmos. Oceans, 2, 341-381, https:// doi.org/10.1016/0377-0265(78)90002-7.

Hayashi, Y., 1980: Estimation of nonlinear energy transfer spectra by the cross-spectral method. J. Atmos. Sci., 37, 299-307, https://doi.org/10.1175/1520-0469(1980)037<0299: EONETS $>2.0 . \mathrm{CO} ; 2$.

Hogg, A. M., and J. R. Blundell, 2006: Interdecadal variability of the Southern Ocean. J. Phys. Oceanogr., 36, 1626-1645, https://doi.org/10.1175/JPO2934.1.

_ , W. K. Dewar, P. D. Killworth, and J. R. Blundell, 2003: A quasi-geostrophic coupled model (Q-GCM). Mon. Wea. Rev., 131, 2261-2278, https://doi.org/10.1175/1520-0493(2003)131<2261: $\mathrm{AQCMQ}>2.0 . \mathrm{CO} ; 2$.

,,--- , and -2006 : Decadal variability of the midlatitude climate system driven by the ocean circulation. J. Climate, 19, 1149-1166, https://doi.org/10.1175/JCLI3651.1.

, J. R. Blundell, W. K. Dewar, and P. D. Killworth, 2014: Formulation and users' guide for Q-GCM version 1.5.0. $56 \mathrm{pp}$., http://q-gcm.org/downloads/q-gcm-v1.5.0.pdf.

Hua, B. L., and D. B. Haidvogel, 1986: Numerical simulations of the vertical structure of quasi-geostrophic turbulence. J. Atmos. Sci., 43, 2923-2936, https://doi.org/10.1175/15200469(1986)043<2923:NSOTVS > 2.0.CO;2.

Huck, T., O. Arzel, and F. Sévellec, 2015: Multidecadal variability of the overturning circulation in presence of eddy turbulence. J. Phys. Oceanogr., 45, 157-173, https://doi.org/10.1175/JPOD-14-0114.1.

Kirtman, B. P., N. Perlin, and L. Siqueira, 2017: Ocean eddies and climate predictability. Chaos, 27, 126902, https://doi.org/ 10.1063/1.4990034.

Kiss, A. E., and L. M. Frankcombe, 2016: The influence of periodic forcing on the time dependence of western boundary currents: Phase locking, chaos, and mechanisms of low-frequency variability. J. Phys. Oceanogr., 46, 1117-1136, https://doi.org/ 10.1175/JPO-D-15-0113.1.

Kravtsov, S., P. Berloff, W. K. Dewar, M. Ghil, and J. C. McWilliams, 2006: Dynamical origin of low-frequency variability in a highly nonlinear midlatitude coupled model. J. Climate, 19, 6391-6408, https://doi.org/10.1175/JCLI3976.1. 
LaCasce, J. H., 2002: On turbulence and normal modes in a basin. J. Mar. Res., 60, 431-460, https://doi.org/10.1357/ 002224002762231160.

Larichev, V. D., and I. M. Held, 1995: Eddy amplitudes and fluxes in a homogeneous model of fully developed baroclinic instability. J. Phys. Oceanogr., 25, 2285-2297, https://doi.org/ 10.1175/1520-0485(1995)025<2285:EAAFIA $>2.0 . C O ; 2$.

Longuet-Higgins, M. S., 1964: Planetary waves on a rotating sphere. Proc. Roy. Soc. London, 279A, 446-473, https:// doi.org/10.1098/rspa.1964.0116.

Minobe, S., A. Kuwano-Yoshida, N. Komori, S.-P. Xie, and R. J. Small, 2008: Influence of the Gulf Stream on the troposphere. Nature, 452, 206-209, https://doi.org/10.1038/nature06690.

Morten, A. J., 2015: Spatio-temporal spectra and spectral transfers in fluid dynamics. Ph.D. thesis, University of Michigan, 133 pp., https://doi.org/2027.42/116695.

O'Rourke, A. K., B. K. Arbic, and S. M. Griffies, 2018: Frequencydomain analysis of atmospherically forced versus intrinsic ocean surface kinetic energy variability in GFDL's CM2-O model hierarchy. J. Climate, 31, 1789-1810, https://doi.org/ 10.1175/JCLI-D-17-0024.1.

Penduff, T., M. Juza, B. Barnier, J. Zika, W. K. Dewar, A.-M. Treguier, J.-M. Molines, and N. Audiffren, 2011: Sea level expression of intrinsic and forced ocean variabilities at interannual time scales. J. Climate, 24, 5652-5670, https:// doi.org/10.1175/JCLI-D-11-00077.1.

Quattrocchi, G., S. Pierini, and H. A. Dijkstra, 2012: Intrinsic lowfrequency variability of the Gulf Stream. Nonlinear Processes Geophys., 19, 155-164, https://doi.org/10.5194/npg-19-155-2012.

Renault, L., M. J. Molemaker, J. Gula, S. Masson, and J. C. McWilliams, 2016: Control and stabilization of the Gulf Stream by oceanic current interaction with the atmosphere. J. Phys. Oceanogr., 46, 3439-3453, https://doi.org/10.1175/ JPO-D-16-0115.1.

Salmon, R., 1978: Two-layer quasi-geostrophic turbulence in a simple special case. Geophys. Astrophys. Fluid Dyn., 10, 25 52, https://doi.org/10.1080/03091927808242628.

_ 1980: Baroclinic instability and geostrophic turbulence. Geophys. Astrophys. Fluid Dyn., 15, 167-211, https://doi.org/ 10.1080/03091928008241178.

Saltzman, B., 1957: Equations governing the energetics of the large scales of atmospheric turbulence in the domain of wave number. J. Meteor., 14, 513-523, https://doi.org/10.1175/15200469(1957)014<0513:EGTEOT>2.0.CO;2.

Scott, R. B., and F. Wang, 2005: Direct evidence of an oceanic inverse kinetic energy cascade from satellite altimetry. J. Phys. Oceanogr., 35, 1650-1666, https://doi.org/10.1175/JPO2771.1.

Sérazin, G., T. Penduff, S. Grégorio, B. Barnier, J.-M. Molines, and L. Terray, 2015: Intrinsic variability of sea level from global ocean simulations: Spatiotemporal scales. J. Climate, 28, 4279_ 4292, https://doi.org/10.1175/JCLI-D-14-00554.1.

, - - B. Barnier, J.-M. Molines, B. K. Arbic, M. Müller, and L. Terray, 2018: Inverse cascades of kinetic energy as a source of intrinsic variability: A global OGCM study. J. Phys. Oceanogr., 48, 1385-1408, https://doi.org/10.1175/JPO-D-170136.1.

Sheng, J., and Y. Hayashi, 1990a: Estimation of atmospheric energetics in the frequency domain during the FGGE year. J. Atmos. Sci., 47, 1255-1268, https://doi.org/10.1175/15200469(1990)047<1255:EOAEIT>2.0.CO;2.

——, and ——, 1990b: Observed and simulated energy cycles in the frequency domain. J. Atmos. Sci., 47, 12431254, https://doi.org/10.1175/1520-0469(1990)047<1243: OASECI $>2.0 . \mathrm{CO} ; 2$

Shevchenko, I., P. Berloff, D. Guerrero-López, and J. E. Roman, 2016: On low-frequency variability of the midlatitude ocean gyres. J. Fluid Mech., 795, 423-442, https://doi.org/10.1017/ jfm.2016.208.

Simonnet, E., and H. A. Dijkstra, 2002: Spontaneous generation of low-frequency modes of variability in the wind-driven ocean circulation. J. Phys. Oceanogr., 32, 1747-1762, https://doi.org/ 10.1175/1520-0485(2002)032<1747:SGOLFM>2.0.CO;2.

Small, R., and Coauthors, 2008: Air-sea interaction over ocean fronts and eddies. Dyn. Atmos. Oceans, 45, 274-319, https:// doi.org/10.1016/j.dynatmoce.2008.01.001.

Steinberg, H. L., A. Wiin-Nielsen, and C.-H. Yang, 1971: On nonlinear cascades in large-scale atmospheric flow. J. Geophys. Res., 76, 8629-8640, https://doi.org/10.1029/JC076I036P08629.

Stull, R. B., 1988: An Introduction to Boundary Layer Meteorology. Kluwer Academic, 670 pp.

Tennekes, H., and J. Lumley, 1972: A First Course in Turbulence. MIT Press, 300 pp.

Vallis, G. K., 2006: Atmospheric and Oceanic Fluid Dynamics. Cambridge University Press, 745 pp.

von Storch, J.-S., H. Sasaki, and J. Marotzke, 2007: Wind-generated power input to the deep ocean: An estimate using a $1 / 10^{\circ}$ general circulation model. J. Phys. Oceanogr., 37, 657-672, https://doi.org/10.1175/JPO3001.1.

Wilson, C., 2016: Does the wind systematically energize or damp ocean eddies? Geophys. Res. Lett., 43, 12 538-12 542, https:// doi.org/10.1002/2016GL072215.

_ C. W. Hughes, and J. R. Blundell, 2015: Forced and intrinsic variability in the response to increased wind stress of an idealized Southern Ocean. J. Geophys. Res. Oceans, 120, 113-130, https://doi.org/10.1002/2014JC010315.

Zhai, X., H. L. Johnson, and D. P. Marshall, 2010: Significant sink of ocean-eddy energy near western boundaries. Nat. Geosci., 3, 608-612, https://doi.org/10.1038/ngeo943. 